\title{
Unified treatment of electron-ion recombination in the close-coupling approximation
}

\author{
Sultana N. Nahar and Anil K. Pradhan \\ Department of Astronomy, The Ohio State University, Columbus, Ohio 43210
}

(Received 2 September 1993)

\begin{abstract}
A unified treatment for electron-ion recombination has been developed and applied to detailed calculations for cross sections and total recombination rate coefficients for a number of atoms and ions. The $a b$ initio calculations are carried out in the close-coupling approximation employing the $R$-matrix method for the bound and the continuum states of the electron-ion system. All final states of the recombined system are taken into account and the present method subsumes both the radiative and the dielectronic recombination ( $R R$ and $D R$ ) processes over all energy and temperature ranges of practical importance. Recombined states of the electron-ion system are divided into two groups: (a) $n \leq n_{0}$ and (b) $n_{0}<n \leq \infty$. Photoionization cross sections are calculated for all bound states of group (a), typically a few hundred bound states, referred to as low- $n$ states, including a detailed energy resolution of the several infinite series of autoionizing resonances converging onto the various excited states of the core ion included in the close-coupling expansion. High- $n$ states of group (b) are treated primarily through the theory of DR developed by Bell and Seaton [J. Phys. B 18, 1589 (1985)]. DR collision strengths with detailed resonance structures are presented. The detailed and the resonance-averaged $\mathrm{DR}$ collision strengths show characteristic peaks at the target thresholds of the core ion corresponding to dipoleallowed transitions. Individual bound states of the $e+$ ion system with dominant contribution to lowenergy recombination are described. The present results demonstrate the importance of (i) recombinations to excited states (particularly the metastable states), and (ii) low-energy autoionizing resonances, both of which result in large contributions to effective electron-ion recombination. The individual contributions of the excited bound states of the $e+$ ion system are calculated and their relative contribution to the total are discussed. The general pattern of the recombination rate, as a function of electron temperature, is studied along an isoelectronic sequence. It is found that while the low-energy (temperature) recombination increases with ion charge $z$, the relative high-energy (temperature) contribution to the total decreases; i.e., viewed as independent processes, the RR part increases while the DR decreases with $z$. Total recombination rate coefficients for several atoms and ions (C II, S II, C II, N II, O III, F IV, Ne v, and $\mathrm{Si}$ IX) are obtained over the entire temperature range of possible interest in applications. Comparisons are made with earlier works on $R R$ and $D R$.
\end{abstract}

PACS number(s): 34.80.Kw, 32.80.Dz, 32.80.Fb

\section{INTRODUCTION}

Recombination of free electrons with ions is the mechanism whereby a plasma establishes ionization equilibrium and is of interest in many diverse laboratory and astrophysical sources. Electron-ion recombination is generally treated as two separate and independent processes: radiative and dielectronic recombination ( $R R$ and $D R$ ). The former refers to recombination of free electrons with an ion through a structureless continuum, while the latter refers to recombination through the autoionizing resonances. In the latter, DR process, the incident electron is first captured into a doubly excited state of the $e+$ ion system and later undergoes radiative decay to a pure bound state of the recombined system. Calculations for the RR consider the inverse photoionization process, usually of the ground state and excited states in relatively simple approximations that do not explicitly account for autoionization. On the other hand, although the DR process involves competing processes of radiative stabilization and autoionization, it is usually treated in the individual resonance approximation involving the calculation of radiative and autoionization probabilities independent of each other [1]. Normally the DR process is important at higher electron energies, and temperatures, since it involves electrons energetic enough to excite the target states of the ion that support the Rydberg series of resonances. It is sometimes also necessary to consider the DR process in the low-energy region in case of resonances close to the ionization threshold which may contribute considerably to recombination [2]; it is thus critical to obtain the positions of such resonances accurately. Many different approximations of varying accuracy, ranging from central field models and atomic structure calculations to more sophisticated distorted wave and Hartree-Fock approximations are employed for these calculations. Total electron-ion recombination rates are then obtained by an addition of the rates for the RR and the DR processes, both the low-temperature and the high-temperature DR rates need to be considered in practical applications [3]. Not only does this imply independent treatment of physical processes that are inextricably linked, but the available data may be of inconsistent accuracy and need to be obtained from different sources for the calculation of total, effective recombination rates of interest in plasma sources. 
In earlier works (Nahar and Pradhan [4,5]; hereafter NP1 and NP2) the authors reported some of the first results on a computationally unified, $a b$ initio treatment of electron-ion recombination. In NP1 and NP2 we described some aspects of what are fairly extensive calculations, and it is the purpose of this report to describe the method in detail with applications to several atomic systems and comparisons with available results on RR and DR. The method forms an extension of the closecoupling (CC) approximation, hitherto employed for electron-ion scattering and photoionization, to electronion recombination. Part of the work may also be regarded as an extension of the Opacity Project [6,7] (hereafter OP), which entails extensive calculations of photoionization cross sections of all bound states of an atom or ion with $n \leq n_{0}$ [group (a) or low- $n$ states referred to earlier]. Under the Opacity Project the photoionization cross sections of nearly 200 atoms and ions, primarily of astrophysical abundant elements, have been computed. However all photoionization calculations reported in the present work have not been reported previously, with consideration of a larger number of $L S$ bound states and, as explained later, involve higher resolution in terms of energy or the effective quantum number to delineate the resonance structures as completely as feasible. Another part of the work is the application of the $a b$ initio theory of DR developed by Bell and Seaton [8] (hereafter BS), that is employed for the calculation of recombination through high- $n$ resonance states, $n_{0}<n \leq \infty$ [group (b)], to the recombined ion. The BS theory accounts for interactions between the radiation field and the wave functions for the $e+$ ion system; the latter are assumed to have been obtained by allowing for resonances but neglecting radiation damping. The detailed DR collision strengths, with resonances in the emitted photon spectrum, are calculated for the first time. Sample results are presented for a number of atoms and ions: $\mathrm{C}$ II, S II, C I, N II, O III, F IV, Ne V, and Si IX. The complete results in numerical form will be presented elsewhere. Several interesting consequences of the BS theory are described here. Previous calculations for DR rate coefficients obtained by other workers are compared with the present ones obtained with the BS theory, as well as with the total recombination rate coefficients.

Some earlier works have sought to develop unified theories of electron-ion recombination $[9,10]$, which, however, are confined to the individual resonance approximation. Also, no detailed results with the application of the earlier treatments have yet been obtained. We employ the term "unified" in a computational sense, in that all significant contributions to electron-ion recombination have been considered using a basis set of coupled eigenfunctions in an $a b$ initio manner. The present treatment extends to all electron energies likely to contribute to recombination, and therefore a single, total recombination rate coefficient for a given atom or ion is obtained at all temperatures of possible interest (results are presented in the range $10<T<10^{9} \mathrm{~K}$ ). The method involves a number of steps, with large-scale calculations for each atom or ion, and it is the purpose of this paper to describe in detail these steps and the results obtained.

\section{THEORY}

We consider the infinity of final states of the recombined $e+$ ion system and the processes that lead to recombination into those states. For low-lying bound states of the $e+$ ion system, the probabilities of recombination are related to the cross sections for the inverse process of photoionization, including autoionizing resonances, which therefore account for both the RR and the DR processes in that recombinations through the continua as well as the resonances are included. It is important, however, to ensure that the subset of low-lying states, low- $n$ states of group (a), is complete in the following sense: all bound states up to $n \leq n_{0}$ must be included, as well as all autoionizing resonances in the photoionization cross sections up to $n \leq n_{0}$ must be resolved. Thus recombination through a given autoionizing resonance with $n \leq n_{0}$ must end up in a bound state included in group (a). One usually thinks of the recombining electron as a "spectator" such that the initial and the final quantum numbers in the resonance and the bound $e+$ ion state remain the same. However, if the subset of total $e+$ ion symmetries is complete in terms of the total angular momenta of contributing states, then the radiative decay of a resonant state to a bound state of different symmetry (due to $l$-changing under dipole selection rules) is also accounted for, since the particular bound state will have been included in group (a).

Figure 1 represents schematically the electron-ion recombination process as viewed in terms of the closecoupling method, with a number of states of the "target" ion prior to recombination with the incident electrons and final bound states of the recombined $e+$ ion system.

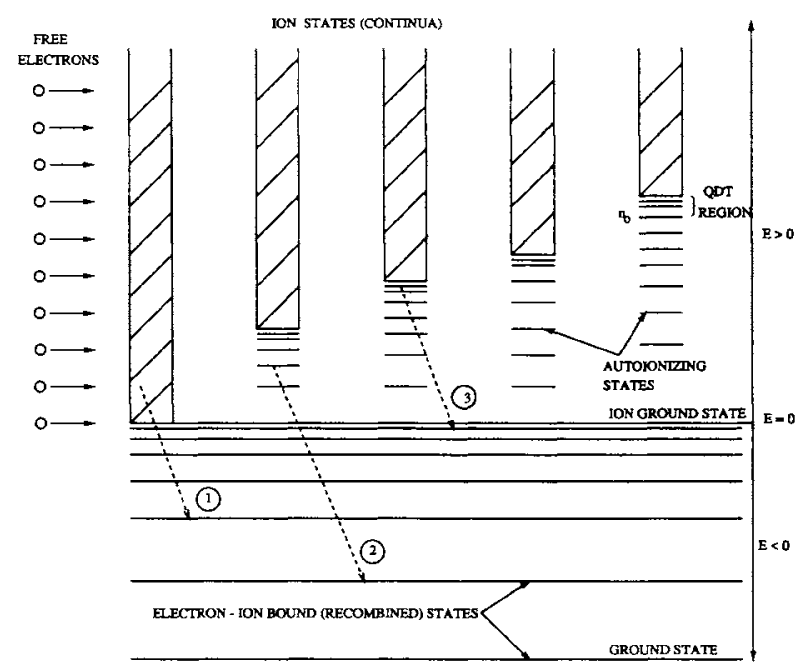

FIG. 1. Schematic diagram for electron-ion recombination. The infinite series of autoionizing resonances converging onto the various target states are in the region $E>0$, while recombined states are in the $E<0$ region. Broken lines with arrows represent photon emission during recombination: (1) represents recombination through the continua, (2) through a low- $n$ autoionizing state with possibly large interaction with the continua, and (3) through a high- $n$ autoionizing state with negligible continuum contribution. 
The coupled eigenfunction expansion represents the continua and the infinite series of autoionizing resonances converging on to the individual target states. In this region $(E>0)$ the total energy of the $e+$ ion system and continuum wave functions must be calculated. The final states are the bound states of the $e+$ ion system with $E<0$. The $\mathrm{CC}$ expansion in terms of the target eigenfunction $\psi_{i}$ is usually written as

$$
\Psi(e+\text { ion })=\sum_{i} \psi_{i} \theta_{i}+\sum_{j} c_{j} \Phi_{j},
$$

which describes both the continuum and the bound states of the $e+$ ion system. The $\theta_{i}$ refers to the free electron wave functions in the free channels $i$ and the $\Phi_{j}$ refer to $L^{2}$-type bound channel functions $j$ important for shortrange correlation (the $c_{j}$ are variational coefficients). If the interaction between the embedded resonances and the background continua is substantial, in the region of recombination through the low- $n$ states $\left(n \leq n_{0}\right)$, then a distinction between RR and DR may not be made. An example of this contribution, for O III, is discussed in NP2. It is therefore necessary to calculate all the detailed resonance profiles, as present in the photoionization cross sections, reflecting the magnitude of this interaction. However, in the region of recombination through the high- $n$ resonances, just below the target thresholds included in the coupled channel scheme, the resonances are narrow and dense and the background continuum contribution is very small; DR dominates the recombination process for high- $n$ resonances. Even so, as we show later in our results, the contribution from the high-energy regions of the photoionization cross sections of low- $n$ bound states, at energies and temperatures where DR is predominant, is small but not insignificant. Also, we find that the background continuum contribution for recombination to high- $n$ bound states does not usually exceed $1 \%$ of the total. Depending upon the choice of $n_{0}$, the small energy region, $n_{0} \leq n \leq \infty$, below each threshold of convergence, is designated as the QDT (quantum-defect theory) region where we use the $\mathrm{BS}$ theory which gives QDT expressions for the calculation of DR probabilities.

\section{A. Recombination to low- $n$ states}

The present work considers contributions from the detailed photoionization cross sections that include autoionizing resonances, of all Ls bound states of the $e+$ ion system with $n \leq n_{0}$ and $l \leq l_{\max }$. (We usually choose $n_{0}=10$ and $l_{\max }=5$ to 9 - the previous OP calculations for the second row elements include $l_{\max }=3$ ). In the discussion throughout this report we have used the general notation $n$ and $n_{0}$ to indicate only the principal quantum number. However, in the computations we employ the corresponding effective quantum number $v$ both as a discrete and a continuous variable, in referring to pure bound and autoionizing states, respectively. The bound states correspond to the "zeroes" in the $e+$ ion Hamiltonian, which are so determined as to ensure that all bound states of the $e+$ ion system are found, including the equivalent electron states. All autoionizing resonances up to effective quantum number $v=10.0$ are resolved. Gailitis averaging [11] is carried out over the autoionizing resonances in the region $10.0 \leq v \leq \infty$ (QDT region). Given the detailed photoionization cross section $\sigma_{\mathrm{PI}}$, as a function of the photoelectron energy, the recombination cross section is obtained using the Milne relation $[3]$ as

$$
\sigma_{\mathrm{RC}}=\sigma_{\mathrm{PI}} \frac{g_{i}}{g_{j}} \frac{h^{2} \omega^{2}}{4 \pi^{2} m^{2} c^{2} v^{2}},
$$

where $g_{i}, g_{j}$ are the statistical weights of the initial ion and the residual ion, respectively, $v$ is the velocity of the photoelectron, and $\omega$ is the photon angular frequency. Assuming a Maxwellian distribution of electrons at a temperature $T$, the contribution to the recombination rate coefficient from the low- $n$ states is

$$
\begin{aligned}
\alpha\left(T ; n \leq n_{0}\right)=\sum_{i_{b}}^{N_{\text {bnd }}} & \frac{g_{i}}{g_{j}} \frac{2}{k T \sqrt{2 \pi m^{3} k T c^{2}}} \\
& \times \int_{0}^{\infty} E^{2} \sigma_{\mathrm{PI}}\left(i_{b} ; \varepsilon\right) e^{-\varepsilon / k T} d \varepsilon,
\end{aligned}
$$

where $E=h \omega / 2 \pi=\varepsilon+I_{p}, \varepsilon$ is the photoelectron energy, and $I_{p}$ is the ionization potential. The sum over the bound states $i_{b}$ extends up to all bound states $N_{\text {bnd }}$ of the $e+$ ion system. Typically $N_{\text {bnd }}$ is of the order of a few hundred for the atoms and ions considered in this work.

\section{B. Recombination to high- $n$ states}

For the high- $n$ states, $n_{0}<n \leq \infty$, where recombination proceeds predominantly through DR, we employ the precise BS theory to obtain DR probabilities and collision strengths in detail as a continuous function of energy. The resonance structures are delineated and resonanceaveraged values are calculated using analytical and numerical averaging procedures. Exactly the same eigenfunction expansion is used in the continuum state calculations for $D R$ as in the photoionization calculations. Using the BS theory the coupled channel eigenfunctions allowing for autoionization resonances, but neglecting radiative decays, have been obtained. The expressions for the scattering matrices for the detailed and the resonance-averaged DR collision strength are thus related to multichannel quantum-defect theory (MCQDT), analogous to those for electron-ion scattering. We describe the DR collision theory briefly below.

\section{The generalized electron-photon scattering matrix}

Including radiative interactions in an $a b$ initio manner in the interaction Hamiltonian for the $e+$ ion system, Davies and Seaton [12] obtain a general scattering matrix $\mathcal{S}$ that is partitioned as

$$
\mathcal{S}=\left[\begin{array}{ll}
\mathcal{S}_{e e} & \mathcal{S}_{e p} \\
\mathcal{S}_{p e} & \mathcal{f}_{p p}
\end{array}\right],
$$

where $\mathcal{S}_{e e}$ is the matrix for electron scattering including radiation damping; $\mathcal{S}_{p e}$ is the matrix for electron capture followed by radiative decay with the emission of a pho- 
ton; $\S_{e p}$ that for the inverse process of photoionization; and $\mathcal{S}_{p p}$ is the matrix for photon-photon scattering (not explicitly considered by the BS theory). In the absence of interaction with the radiation field $\delta_{e e}$ is the usual scattering matrix \&. The unitarity condition for $\delta$ reflects the conservation of both the incident electron and the emitted photon flux [12], i.e.,

$$
\mathcal{e}_{e e}^{\dagger} \mathcal{e}_{e e}+\mathcal{s}_{p e}^{\dagger} \mathcal{\rho}_{p e}=1 \text {. }
$$

According to MCQDT $[8,13]$, the electron scattering matrix $\delta_{e e}$ may be again partitioned into submatrices of open and closed channels in the energy region below threshold, i.e., in the QDT region. In this energy region the analytic continuation of $\&_{e e}$ may be expressed in terms of the matrix $\chi$ as $\chi_{o o}, \chi_{o c}, \chi_{c o}$, and $\chi_{c c}$, where $o$ denotes the open and $c$ the closed channels. The open channels are those that are accessible to the incident electron for excitation of a target state in that channel; a closed channel refers to electron energies below an inaccessible target threshold. A given Rydberg series of resonances, converging onto a target threshold $S_{t} L_{t}$, corresponds to the closed channel $\left(S_{t} L_{t}\right) \varepsilon l$, where $\varepsilon=-1 / v^{2}$, $v$ is the effective quantum number associated with the resonance series. Successive resonances, $S_{t} L_{t} n l$, in the series correspond to $\Delta v=1$. The continuous variable $v$ may be related to the electron energy $E$, relative to the target threshold $S_{t} L_{t}$, by

$$
E=E\left(S_{t} L_{t}\right)-z^{2} / v^{2}
$$

where $z$ is the ion charge. We further subdivide the group of closed channels according to the target terms $S_{t} L_{t}$ they belong to: the closed channels corresponding to Rydberg series of resonances in the QDT region with $v>v_{\max }\left(n_{0}\right)$ are called "weakly closed channels," and the ones with $v<v_{\max }$, that belong to higher thresholds, are labeled as "strongly closed." The weakly closed channels belong to the nearest target threshold of convergence and the channel functions are rapidly oscillating since the number of nodes, corresponding to the narrow high- $n$ resonances, increases as the energy approaches the target threshold where the channels become open. The Gailitis averaging procedure entails an analytic average over the narrow resonances corresponding to the weakly closed channels. The series of resonances corresponding to strongly closed channels are broader and may overlap with the resonances due to weakly closed channels. Also, the target thresholds may lie close enough in energy so that the QDT regions may overlap. These cases present some difficulties in practical computations that we discuss later.

The expression given by the BS theory for $\delta_{e e}$ in terms of its analytic continuation $\chi$ is

$$
\mathcal{f}_{e e}=\chi_{\infty o}-\chi_{o c}\left[\chi_{c c}-g(v) \exp (-2 i \pi v)\right]^{-1} \chi_{c o},
$$

where $g(v)=\exp \left(\pi v^{3} A_{r} / z^{2}\right) ; A_{r}$ is the sum of all possible radiative decay probabilities for the available decay routes from a given excited state of the target ion. The outer electron is treated as a "spectator," in a high- $n$ resonance state, interacting but weakly with the core.

\section{DR transition probability and collision strength}

The unitarity condition yields the DR probability, for an entrance or incident open channel $\alpha$, as

$$
P_{\alpha}=\left(1-\rho_{e e}^{\dagger} \rho_{e e}\right)_{\alpha \alpha}
$$

or

$$
P_{\alpha}=1-\sum_{\alpha^{\prime}}\left|\mathcal{S}_{\alpha \alpha^{\prime}}\right|^{2},
$$

where $\alpha^{\prime}$ goes over all the open channels.

The electron flux trapped in the closed channel resonances may decay radiatively to bound states of the $e+$ ion system. In MCQDT if one considers the closed channels to be degenerate then it is useful to diagonalize the $\chi$ matrix as

$$
\chi_{c c} \mathbf{N}=\mathbf{N} \chi_{c c},
$$

where $\chi_{c c}$ is a diagonal matrix and $\mathbf{N}$ is the diagonalizing matrix with $\mathbf{N}^{T} \mathbf{N}=1$. In terms of $\mathbf{N}$ we write $\chi_{o c}^{\prime}=\chi_{o c} \mathbf{N}$ and $\boldsymbol{\chi}_{c o}^{\prime}=\mathbf{N}^{T} \boldsymbol{\chi}_{c o}$.

The DR probability $P(D R)$ is given by the diagonal elements of the matrix

$$
\begin{aligned}
1-\mathcal{S}^{\dagger} \rho= & G(v) \chi_{o c}\left[\chi_{c c}-g(v) \exp (-2 i \pi v)\right]^{-1} \\
& \times\left[\chi_{c c}^{*}-g(v) \exp (+2 i \pi v)\right]^{-1} \chi_{c o}^{*},
\end{aligned}
$$

where $G(v)=g(v)^{2}-1=\exp \left(2 \pi v^{3} A_{r} / z^{2}\right)-1$. In practical computations use of Eq. (11) can lead to numerical instabilities owing to the fact that $\mathbf{S}^{\dagger} \mathbf{S}$ may be very close to unity. Therefore we rewrite the relevant expressions in the following manner.

Using the diagonal matrix $\chi_{c c}$, which commutes with $\mathbf{N}$, the above matrix can be written as

$$
\begin{aligned}
G(v) \chi_{\alpha c}^{\prime} \mathbf{N}^{T} & {\left[\chi_{c c}-g(v) \exp (-2 i \pi v)\right]^{-1} } \\
& \times\left[\chi_{c c}^{*}-g(v) \exp (+2 i \pi v)\right]^{-1}\left(\mathbf{N} \chi_{c \alpha}^{\prime}\right)^{*} .
\end{aligned}
$$

Expanding in terms of the matrix elements we obtain for the DR probability for the entrance channel $\alpha, P_{\alpha}$,

$$
\begin{aligned}
P_{\alpha}=G(v) \sum_{\gamma}[ & {\left[\sum_{\gamma^{\prime}} \chi_{o \gamma^{\prime}}^{\prime} \mathbf{N}_{\gamma \gamma^{\prime}}\right] } \\
& \times\left[\chi_{\gamma \gamma}-g(v) \exp (-2 i \pi v)\right]^{-1} \\
& \times\left[\chi_{\gamma \gamma}^{*}-g(v) \exp (+2 i \pi v)\right]^{-1} \\
& \left.\times\left[\sum_{\gamma^{\prime}} \chi_{\gamma^{\prime} \alpha}^{\prime *} \mathbf{N}_{\gamma \gamma^{\prime}}^{*}\right]\right]
\end{aligned}
$$

The summations go over the closed channels $\gamma \gamma^{\prime}$ contributing to DR. The sum over the diagonal elements of all open channels linked to the ground state of the target ion gives the probability of DR through radiative transitions between the excited states and the ground state.

As for electron-ion scattering, MCQDT incorporated an analytical averaging procedure due to Gailitis [11]. In an analogous manner the resonance-averaged $D R$ probability may be expressed as [8] 


$$
\left\langle P_{\alpha}\right\rangle=G(v) \sum_{\gamma, \gamma^{\prime}} \frac{\chi_{\alpha \gamma}^{\prime} \chi_{\gamma^{\prime} \alpha}^{\prime *}\left(\mathbf{N}^{T} \mathbf{N}^{*}\right)_{\gamma \gamma^{\prime}}}{G(v)+1-\chi_{\gamma \gamma} \chi_{\gamma^{\prime} \gamma^{\prime}}^{*}},
$$

which for computational convenience we expand into direct and interference terms involving the closed channels as

$$
\begin{aligned}
\left\langle P_{\alpha}\right\rangle=G(v) & {\left[\sum_{\alpha} \frac{\left|\chi_{\alpha \gamma}^{\prime}\right|^{2} \sum_{i}\left|N_{i \gamma}\right|^{2}}{G(v)+1-\left|\chi_{\gamma \gamma}\right|^{2}}\right.} \\
& \left.+2 \sum_{\gamma \neq \gamma^{\prime}} \operatorname{Re}\left[\frac{\chi_{\alpha \gamma}^{\prime} \chi_{\gamma^{\prime} \alpha}^{\prime} \sum_{i} N_{i \gamma} N_{i \gamma^{\prime}}^{*}}{G(\nu)+1-\chi_{\gamma \gamma} \chi_{\gamma^{\prime} \gamma^{\prime}}}\right]\right] .
\end{aligned}
$$

Finally, the DR collision strength is given in terms of the DR probabilities as

$$
\Omega(\mathrm{DR})=\sum_{S, L, \pi} \sum_{\alpha} \frac{1}{2}(2 S+1)(2 L+1) P_{\alpha}^{S L \pi} .
$$

The total $\Omega(\mathrm{DR})$, with detailed resonances or averaged over resonances, is obtained by a complete summation over contributing $S L \pi$ states. The present method is thus complementary, and related through unitarity of the generalized $\&$ matrix to electron-ion scattering when radiation damping is included in the interaction Hamiltonian. At each target threshold $S_{t} L_{t}$, the DR collision strength is related through the resonances of the type $\left(S_{t} L_{t}\right) n l$ to the electron-impact-excitation (EIE) collision strength as

$$
\lim _{n \rightarrow \infty}\langle\Omega(n ; \mathrm{DR})\rangle=\lim _{k^{2} \rightarrow 0} \Omega\left(k^{2} ; \mathrm{EIE}\right) .
$$

It is understood that the EIE transition in the target ion is the same one whose radiative decay contributes to $D R$ through the given resonance series. Equation (17) provides a useful practical check on the DR calculations as its limiting value may be determined from separate calculations for EIE of the target states of the ion. The dimensionless DR collision strength is related to the collision cross section in the usual manner but with a different interpretation. We have

$$
Q\left(k^{2} ; i, j\right)=g_{i} k^{2} \Omega\left(k^{2} ; i, j\right),
$$

where the cross section $Q$ or the collision strength $\Omega$ is obtained for electron excitation of the transitions $i \rightarrow j$, followed by radiative decay of the excited state and the Rydberg resonances converging onto it $\left(k^{2}\right.$ is the incident electron energy). Unlike EIE, we need to consider the contributions to DR from the elastic scattering channels, with resonances due to closed channels belonging to higher thresholds. If we take $i$ to be the ground state, then the DR cross section is calculated for all target transitions from excited states $j$ that are linked radiatively to the ground state. A discussion of EIE including radiation damping of autoionizing resonances, i.e., the effect of DR, has been given by Pradhan [14] and Pradhan and Seaton [15].

\section{COMPUTATIONS}

In principle, the present method for the calculation of total electron-ion recombination cross sections and rate coefficients should be applicable to all atomic systems, subject to the limitations of the $\mathrm{CC}$ approximation. The computation for each atom or ion is divided into three major parts: (i) the atomic structure calculations for the target ion (i.e., the eigenfunction expansion) and the $R$ matrix calculations, (ii) calculations of photoionization cross sections for all bound states in the low- $n$ region, and (iii) DR calculations in the high- $n$ region. The atomic structure calculations in part (i) are carried out employing an extended version of the SUPERSTRUCTURE computer program [16]. The one-electron orbital wave functions of the target ion are obtained in a scaled Thomas-FermiDirac-Amaldi- (TFDA) type potential. The standard CC calculations with the $R$-matrix method have been described in detail within the context of the OP in a number of earlier publications (see, for example, NP1, NP2, and references therein). The photoionization calculations in part (ii) have also been described in earlier works $[6,7]$.

For the present work on recombination, a new code INTFACE, written originally for the calculation of opacities [17], is extended to process the detailed photoionization cross sections and to carry out the integrations for the calculation of the low- $n$ contribution to the recombination rate coefficients. The integration for the rate coefficients, Eq. (3), extends to infinity in energy. For fast convergence at high energy, the following expression for $\alpha(T)$ can be used:

$$
\alpha(T)=-\frac{g_{i}}{g_{j}} \frac{2}{c^{2} \sqrt{2 \pi m^{3} k T}} \int_{1}^{0}[I-k T \ln (x)]^{2} \sigma_{\mathrm{PI}} d x,
$$

where $x=\exp (-\varepsilon / k T)$ which has slow variation at low $T$ and fast variation at high $T$.

The $R$-matrix code for the calculation of the asymptotic wave functions, STGF [7], is modified extensively for the calculations in part (iii), as outlined in the Theory section. In order to determine the generality of the present method, we have carried out full calculations for several selected atoms and ions, including a study of electron-ion recombination along an isoelectronic sequence.

Some general features of the calculations pertain to the standard close-coupling approximation, with the following parameters. The $e+$ ion spin and angular momentum symmetries are given by the set of total $S L \pi$ states considered, which in turn depend on the target states and the free electron partial waves, i.e., on $S_{t} L_{t} \varepsilon l \rightarrow S L \pi$. In the DR calculations all partial waves $l \leq 9$ are included; the contribution from higher ones is negligible. Table I presents the target states in the $\mathrm{CC}$ expansion of the atoms and ions considered here, and Table II presents the radiative transition probabilities $A_{r}$ for the dipoleallowed transitions in the target ion that contribute to DR. The values for $A_{r}$ for the ions in this work are obtained from energy values and oscillator strengths of the target states in the OP data. Experimental energies are used for some ions where available. The DR collision 
TABLE I. Target states, energies (in Ry), and configuration-interaction (CI) expansions. The number of target states $N$ dominated by the spectroscopic configurations is stated as $N$-CC. Except for $\mathrm{CII}$, all the B-like ions have the same $\mathrm{CI}$ expansion.

\begin{tabular}{|c|c|c|c|c|c|c|c|c|}
\hline State & Calc. & Obs. & State & Calc. & Obs. & State & Calc. & Obs. \\
\hline & & & & CII $10-C$ & & & & \\
\hline $2 s^{2} 2 p^{2} P^{o}$ & 0.0 & 0.0 & $2 s 2 p^{22} P$ & 1.0202 & 1.008 & $2 p^{32} D^{o}$ & 1.4166 & 1.3708 \\
\hline $2 s 2 p^{24} P$ & 0.3768 & 0.3918 & $2 s^{2} 3 s^{2} S$ & 1.0615 & 1.0616 & $2 p^{22} P^{o}$ & 1.6207 & 1.5373 \\
\hline $2 s 2 p^{22} D$ & 0.6882 & 0.6824 & $2 s^{2} 3 p^{2} P^{o}$ & 1.2199 & 1.200 & & & \\
\hline $2 s 2 p^{22} s$ & 0.8902 & 0.8789 & $2 p^{34} S^{o}$ & 1.3241 & 1.2939 & & & \\
\hline
\end{tabular}

Spectroscopic: $2 s^{2} 2 p, 2 s 2 p^{2}, 2 s^{2} 3 s, 2 s^{2} 3 p, 2 p^{3}$

Correlation: $2 s^{2} 3 d, 2 s 2 p 3 s, 2 s^{2} 4 s, 2 s^{2} 4 p, 2 s^{2} 4 d, 2 s 2 p 3 p, 2 s 2 p 3 d, 2 s 3 d^{2}, 2 s 3 s 3 p, 2 p^{2} 3 s, 2 p^{2} 3 p, 2 p^{2} 3 d, 2 p^{2} 4 s$, $2 p^{2} 4 p, 2 s 3 p^{2}, 2 s 3 s^{2}, 2 p 3 s 3 p, 2 p 3 s 3 d$

\begin{tabular}{|c|c|c|c|c|c|c|c|c|}
\hline \multicolumn{9}{|c|}{$\mathrm{N}$ III $8-\mathrm{CC}$} \\
\hline $2 s^{2} 2 p^{2} P^{o}$ & 0.0 & 0.0 & $2 s 2 p^{22} S$ & 1.2214 & 1.1927 & $2 p^{32} D^{o}$ & 1.8790 & 1.8505 \\
\hline $2 s 2 p^{24} P$ & 0.5021 & 0.5209 & $2 s 2 p^{22} P$ & 1.3517 & 1.3289 & $2 p^{22} P^{o}$ & 2.1805 & 2.0986 \\
\hline $2 s 2 p^{22} D$ & 0.9281 & 0.9196 & $2 p^{34} S^{o}$ & 1.7191 & 1.7012 & & & \\
\hline \multicolumn{9}{|c|}{$\mathrm{O}_{\text {IV }} 8-\mathrm{CC}$} \\
\hline $2 s^{2} 2 p^{2} P^{o}$ & 0.0 & 0.0 & $2 s 2 p^{22} S$ & 1.5204 & 1.4955 & $2 p^{32} D^{o}$ & 2.3497 & 2.3230 \\
\hline $2 s 2 p^{24} P$ & 0.6293 & 0.6482 & $2 s 2 p^{22} P$ & 1.6659 & 1.6438 & $2 p^{22} P^{\circ}$ & 2.7080 & 2.6315 \\
\hline $2 s 2 p^{22} D$ & 1.1619 & 1.1545 & $2 p^{34} S^{o}$ & 2.1233 & 2.1052 & & & \\
\hline \multicolumn{9}{|c|}{$\mathrm{F} \vee 8-\mathrm{CC}$} \\
\hline $2 s 2 p^{2} P^{o}$ & 0.0 & 0.0 & $2 s 2 p^{22} S$ & 1.8162 & 1.8003 & $2 p^{32} D^{o}$ & 2.8181 & 2.7998 \\
\hline $2 s 2 p^{24} P$ & 0.7558 & 0.7876 & $2 s 2 p^{22} P$ & 1.9761 & 1.9610 & $2 p^{22} P^{o}$ & 3.2328 & 3.1660 \\
\hline $2 s 2 p^{22} D$ & 1.3935 & 1.3932 & $2 p^{34} S^{0}$ & 2.5245 & 2.5211 & & & \\
\hline \multicolumn{9}{|c|}{$\mathrm{Ne}$ vi $8-\mathrm{CC}$} \\
\hline $2 s^{2} 2 p^{2} P^{o}$ & 0.0 & 0.0 & $2 s 2 p^{22} S$ & 2.1140 & 2.1175 & $2 p^{32} D^{o}$ & 3.2938 & \\
\hline $2 s 2 p^{24} P$ & 0.8809 & 0.9029 & $2 s 2 p^{22} P$ & 2.2884 & 2.2747 & $2 p^{22} p^{0}$ & 3.7634 & \\
\hline $2 s 2 p^{22} D$ & 1.6268 & 1.6292 & $2 p^{34} S^{o}$ & 2.9248 & & & & \\
\hline \multicolumn{9}{|c|}{ Si $x \quad 8-C C$} \\
\hline $2 s^{2} 2 p^{2} P^{o}$ & 0.0 & 0.0 & $2 s 2 p^{22} S$ & 3.2832 & 3.3503 & $2 p^{32} D^{0}$ & 5.1384 & 5.2348 \\
\hline $2 s 2 p^{24} P$ & 1.3839 & 1.5117 & $2 s 2 p^{22} P$ & 3.5044 & 3.5774 & $2 p^{22} P^{\circ}$ & 5.8345 & 5.8760 \\
\hline $2 s 2 p^{22} D$ & 2.5390 & 2.6229 & $2 p^{34} S^{o}$ & 4.5098 & 4.6492 & & & \\
\hline
\end{tabular}

Spectroscopic: $2 s^{2} 2 p, 2 s 2 p^{2}, 2 p^{3}$

Correlation: $2 s^{2} 3 s, 2 s^{2} 3 p, 2 s^{2} 3 d, 2 s 2 p 3 s, 2 s 2 p 3 p, 2 s 2 p 3 d, 2 p^{2} 3 s, 2 p^{2} 3 p, 2 p^{2} 3 d, 2 s 3 d^{2}$

\begin{tabular}{|c|c|c|c|c|c|c|c|c|}
\hline \multicolumn{9}{|c|}{ CIII $12-C C$} \\
\hline $2 s^{21} S$ & 0.0 & 0.0 & $2 p^{21} D$ & 1.3945 & 1.3293 & $2 s 3 p^{1} P^{o}$ & 2.3672 & 2.3596 \\
\hline $2 s 2 p^{3} P^{o}$ & 0.4871 & 0.4777 & $2 p^{21} S$ & 1.7563 & 1.6632 & $2 s 3 p^{3} P^{o}$ & 2.3677 & 2.3667 \\
\hline $2 s 2 p{ }^{1} P^{\circ}$ & 1.0393 & 0.9327 & $2 s 3 s^{3} S$ & 2.1603 & 2.1708 & $2 s 3 d^{3} D$ & 2.4622 & 2.4605 \\
\hline $2 p^{23} P$ & 1.2672 & 1.2528 & $2 s 3 s^{1} S$ & 2.2566 & 2.2524 & $2 s 3 d^{1} D$ & 2.5363 & 2.5195 \\
\hline
\end{tabular}

Spectroscopic: $2 s^{2}, 2 s 2 p, 2 p^{2}, 2 s 3 s, 2 s 3 p, 2 s 3 d$

Correlation: $2 p 3 p, 2 s 4 s, 2 s 4 p, 2 p 3 p$

\begin{tabular}{|c|c|c|c|c|c|c|c|c|}
\hline \multicolumn{9}{|c|}{ S III $17-\mathrm{CC}$} \\
\hline $3 p^{23} p$ & 0.0 & 0.0 & $3 p 3 d^{1} D^{o}$ & 0.9508 & 0.9440 & $3 p 3 d{ }^{3} D^{\circ}$ & 1.3926 & 1.3407 \\
\hline $3 p^{21} D$ & 0.1177 & 0.0981 & $3 p 3 d^{3} F^{o}$ & 1.1301 & 1.1163 & $3 p 4 s{ }^{1} P^{o}$ & 1.4235 & 1.3472 \\
\hline $3 p^{2} \mid S$ & 0.2529 & 0.2424 & $3 s 3 p^{31} P^{o}$ & 1.2934 & 1.2419 & $3 s 3 p^{31} D^{o}$ & 1.4397 & 1.3798 \\
\hline $3 s 3 p^{35} S^{0}$ & 0.4815 & 0.5295 & $3 s 3 p^{3}{ }^{3} S^{o}$ & 1.2851 & 1.2530 & $3 p 3 d{ }^{1} F^{o}$ & 1.5125 & 1.4311 \\
\hline $3 s 3 p^{33} D^{\circ}$ & 0.7472 & 0.7609 & $3 p 3 d{ }^{3} P^{\circ}$ & 1.3861 & 1.2991 & $3 p 3 d^{1} P^{0}$ & 1.5806 & 1.4906 \\
\hline $3 s 3 p^{3}{ }^{3} P^{o}$ & 0.8844 & 0.8948 & $3 p 4 s^{3} P^{o}$ & 1.3436 & 1.3341 & & & \\
\hline
\end{tabular}


TABLE II. Transition probabilities ( $A$ values in atomic units) for transitions between the ground state and excited states with dipole-allowed transitions for various target ions. The $A$ values are calculated using energies and dipole oscillator strengths from the OP data. The numbers in brackets denote multiplicative powers of ten.

\begin{tabular}{|c|c|c|c|c|c|c|c|}
\hline Ion & $\begin{array}{l}\text { Ground } \\
\text { state }\end{array}$ & $\begin{array}{l}\text { Excited } \\
\text { states }\end{array}$ & $A_{f i}$ (a.u.) & Ion & $\begin{array}{l}\text { Ground } \\
\text { state }\end{array}$ & $\begin{array}{c}\text { Excited } \\
\text { states }\end{array}$ & $A_{f i}$ (a.u.) \\
\hline C II & ${ }^{2} P^{0}$ & $\begin{array}{l}{ }^{2} D,{ }^{2} S, P \\
{ }^{2} S\end{array}$ & $\begin{array}{l}7.05[-9], 5.80[-8], 1.08[-7] \\
8.56[-9]\end{array}$ & $\begin{array}{l}\mathrm{Ne} \text { VI } \\
\mathrm{SiX}\end{array}$ & $\begin{array}{l}{ }^{2} P^{o} \\
{ }^{2} P^{o}\end{array}$ & $\begin{array}{l}{ }^{2} D,{ }^{2} S,{ }^{2} P \\
{ }^{2} D,{ }^{2} S,{ }^{2} P\end{array}$ & $\begin{array}{l}2.78[-8], 1.32[-7], 2.58[-7] \\
4.80[-8], 2.15[-7], 4.04[-7]\end{array}$ \\
\hline N III & ${ }^{2} P^{0}$ & ${ }^{2} D,{ }^{2} S,{ }^{2} P$ & $1.21[-8], 6.91[-8], 1.39[-7]$ & C III & ${ }^{1} S$ & ${ }^{1} P^{0}, P^{0}$ & $4.55[-8], 8.33[-8]$ \\
\hline O IV & ${ }^{2} \boldsymbol{P}^{o}$ & ${ }^{2} D,{ }^{2} S,{ }^{2} P$ & $1.73[-8], 8.85[-8], 1.78[-7]$ & S III & ${ }^{3} P$ & ${ }^{3} D^{\circ},{ }^{3} P^{o},{ }^{3} S^{o}$ & $1.59[-9], 6.73[-9], 3.5[-7]$, \\
\hline $\mathrm{Fv}$ & ${ }^{2} P^{0}$ & ${ }^{2} D,{ }^{2} S,{ }^{2} P$ & $2.26[-8], 1.11[-7], 2.19[-7]$ & & & ${ }^{3} P^{o}, 3 P^{o},{ }^{3} D^{\circ}$ & $3.12[-8], 2.61[-7], 3.62[-7]$ \\
\hline
\end{tabular}

strengths are calculated in the region $10.0 \leq v \leq \infty$ (QDT region) below each threshold of the target ion. Both the detailed collision strengths with resonance structures and those averaged over resonances are obtained. The peak values of DR collision strengths at the target thresholds are given in Table III to compare with the excitation collision strengths. Numerical integration over both sets of results, convoluted over a Maxwellian distribution, for the DR rate coefficients, $\alpha(n, \mathrm{DR})$, provides a good check on the numerical accuracy of the methods. Although the two sets of results usually agree to within a few percent, the values obtained from the analytical averaging procedure are somewhat more accurate and are adopted. In the photoionization calculations we consider all $L S$ states with $l \leq l_{\max }=5-9$ and $n \leq n_{0}=10$. The corresponding total number of bound states, $N_{\text {bnd }}$, are given in Table IV. Bound states with $l>l_{\max }$ and $n \leq n_{0}$, and $n>n_{0}$ are treated as hydrogenic. Together, the contributions to the recombination rate coefficient from the set of states with $\left[n \leq 10, l>l_{\max }\right]$ and $[n>10]$ is usually less than $1 \%$ for all atoms and ions considered in our work. However, these contributions are included and the total $e+$ ion recombination rate coefficient is obtained as

$$
\begin{aligned}
\alpha_{R}= & {\left[\alpha(n)+\alpha^{\mathrm{H}}\left(n, l>l_{\max }\right)\right]_{n} \leq 10 } \\
& +\left[\alpha(n, \mathrm{DR})+\alpha^{\mathrm{H}}(n)\right]_{n>10},
\end{aligned}
$$

where $\mathbf{H}$ denotes the small continuum contributions from highly excited states obtained using hydrogenic photoionization cross sections [18]. It should be emphasized that the principal quantum number $n$ in Eq. (20) is used only in a generic sense; in practical computations we actually deal with bound states of specific symmetries given by the total $S L \pi$ of the $e+$ ion system.

TABLE III. Excitation collision strengths $\Omega(E I E)$ for dipole-allowed transitions in the target ion from the ground-state and the peak values of DR collision strengths. Both the averaged $(\Omega(D R)\rangle$ and the detailed $\Omega(D R)$ are at threshold energies. The parameter IPERT indicates the inclusion of long-range multipole potentials and high-partial-wave summation for the $\Omega$ (EIE) (see text). Ar-

\begin{tabular}{|c|c|c|c|c|c|c|c|c|c|c|c|}
\hline \multirow{2}{*}{$\begin{array}{c}\text { Target } \\
\text { state }\end{array}$} & \multicolumn{3}{|c|}{$\Omega(\mathrm{EIE})$} & \multirow[t]{2}{*}{$\langle\Omega(D R)\rangle$} & \multirow[t]{2}{*}{$\Omega(\mathrm{DR})$} & \multirow{2}{*}{$\begin{array}{c}\text { Target } \\
\text { state }\end{array}$} & \multicolumn{3}{|c|}{$\Omega($ EIE $)$} & \multirow[t]{2}{*}{$\langle\Omega(D R)\rangle$} & \multirow[t]{2}{*}{$\Omega(\mathrm{DR})$} \\
\hline & IPERT $=0$ & 1 & 2 & & & & IPERT $=0$ & 1 & 2 & & \\
\hline & C I: [C II t & rget $\mathrm{gr}$ & ad state: & $\left.2 s^{2} 2 p^{2} P^{o}\right]$ & & & {$[\mathbf{N}$ III t } & get gro & d state: & $\left.2 s^{2} 2 p^{2} P^{o}\right]$ & \\
\hline $2 s 2 p^{22} D$ & 5.931 & 5.718 & 5.718 & 6.028 & 5.932 & $2 s 2 p^{22} D$ & 5.088 & 5.834 & 5.834 & 5.110 & 5.086 \\
\hline $2 s 2 p^{22} S$ & 2.929 & 3.062 & 3.062 & 2.929 & 2.929 & $2 s 2 p^{22} S$ & 2.067 & 2.008 & 2.008 & 2.067 & 2.067 \\
\hline $2 s 2 p^{22} P$ & 4.870 & 5.443 & 5.443 & 4.891 & 4.870 & $2 s 2 p^{22} P$ & 9.291 & 8.836 & 8.836 & 9.354 & 9.292 \\
\hline $2 s^{2} 3 s^{2} S$ & 0.885 & 0.607 & 0.607 & 0.8501 & 0.850 & & & & & & \\
\hline
\end{tabular}
rows indicate that the threshold value has been added to the next higher threshold value.

O III: [O IV target ground state: $\left.2 s^{2} 2 p^{2} P^{o}\right]$

$\begin{array}{llllll}2 s 2 p^{22} D & 4.060 & 5.281 & 5.284 & 4.337 & 4.07 \\ 2 s 2 p^{22} S & 1.962 & 2.204 & 2.204 & 1.963 & 1.963\end{array}$

$\begin{array}{llllll}2 s 2 p^{2} S & 1.962 & 2.204 & 2.204 & 1.963 & 1.963 \\ 2 s 2 p^{22} P & 6.091 & 6.856 & 6.856 & 6.129 & 6.110\end{array}$

Nev: [Nevi target ground state: $2 s^{2} 2 p^{2} P^{o}$ ]

$\begin{array}{llllll}2 s 2 p^{22} D & 3.806 & 3.368 & 3.387 & 3.811 & 3.971 \\ 2 s 2 p^{22} S & 1.825 & 1.821 & 1.821 & 4.351 & 4.349 \\ 2 s 2 p^{22} P & 6.206 & 5.749 & 5.751 & 6.220 & 6.246\end{array}$

C II: [C III target ground state: $2 s^{21} \mathrm{~S}$ ]

$\begin{array}{lllll}2 s 2 p^{1} P^{o} & 3.147 & 3.336 & 3.336 & 3.153\end{array}$

$\begin{array}{lllll}2 s 3 p^{1} P^{\circ} & 0.0917 & 0.0846 & 0.0846 & 0.0987\end{array}$

$2 s 2 p^{22} D$
$2 s 2 p^{22} S$
$2 s 2 p^{22} P$

Fiv: $[\mathrm{Fv}$ target ground state: 4.058 1.839

4.100

2.868

4.109

7.107

6.269

2.868

6.270

Si IX: [Six target ground state:

$2 s 2 p^{22} D$
$2 s 2 p^{2} S$
$2 s 2 p^{2} P$

1.721
0.704
3.373

1.832
0.661

1.898

3.228

0.661

3.242

$2 s^{2} 2 p^{2} P^{0}$
4.163
1.907
7.139

4.077

1.907

7.106

$\left.2 s^{2} 2 p^{2} P^{o}\right]$

1.722

$\downarrow$
4.080

1.911

$\downarrow$

4.077

3.147
0.0917

SII: [S III target ground state: $3 s^{2} 3 p^{23} P$ ]

$\begin{array}{llllll}3 s 3 p^{3}{ }^{3} D^{\circ} & 7.984 & 7.829 & 7.829 & 7.809 & 9.20\end{array}$

$\begin{array}{llllll}3 s 3 p^{3}{ }^{3} P^{o} & 4.734 & 4.488 & 4.488 & 4.757 & 4.718\end{array}$

$\begin{array}{llllll}3 s 3 p^{3}{ }^{3} S^{\circ} & 5.99 & 5.183 & 5.183 & 5.99 & 5.99\end{array}$

$\begin{array}{llllll}3 p 4 s^{3} P^{\circ} & 8.922 & 9.557 & 9.551 & \downarrow & \downarrow\end{array}$

$\begin{array}{llllll}3 p 3 d^{3} P^{\circ} & 1.430 & 1.411 & 1.411 & 10.87 & 10.67\end{array}$

\begin{tabular}{llllll}
$3 p 3 d^{3} D^{o}$ & 16.67 & 17.83 & 17.83 & 18.09 & 16.67 \\
\hline
\end{tabular} 
TABLE IV. Partial recombination rate coefficients from the dominant states, in order of their contributions to low- $n \alpha_{R}(T)$ (in $\mathrm{cm}^{3} / \mathrm{sec}$ ) at temperatures 100,1000 , and $10000 \mathrm{~K} . N_{\text {bnd }}$ corresponds to the total number of bound states for which the detailed photoionization cross sections have been calculated and included in $\alpha_{R}(T)$. The sum and the percentage contribution of these states to the total $\alpha_{R}(T)$ is given at the end of each column. The numbers in brackets denote multiplicative powers of ten.

\begin{tabular}{|c|c|c|c|c|c|}
\hline \multicolumn{2}{|c|}{$T=100 \mathrm{~K}$} & \multicolumn{2}{|c|}{$T=1000 \mathrm{~K}$} & \multicolumn{2}{|c|}{$T=10000 \mathrm{~K}$} \\
\hline State & Coefficient & State & Coefficient & State & Coefficient \\
\hline \multicolumn{6}{|c|}{ C I: $\quad N_{\text {bnd }}=217$} \\
\hline $2 p^{23} P$ & $2.54[-12]$ & $2 p^{23} P$ & $8.11[-13]$ & $2 p^{23} P$ & $2.82[-13]$ \\
\hline $2 s 2 p^{35} S^{0}$ & $9.86[-13]$ & $2 s 2 p^{35} S^{0}$ & $3.15[-13]$ & $2 p^{21} D$ & $1.64[-13]$ \\
\hline $2 p^{21} D$ & $7.73[-13]$ & $2 p^{21} D$ & $2.50[-13]$ & $2 s 2 p^{35} S^{o}$ & $1.09[-13]$ \\
\hline $2 p 3 d^{3} F^{o}$ & $1.51[-13]$ & $2 p 3 d^{3} F^{o}$ & $4.55[-14]$ & $2 p^{21} S$ & $1.52[-14]$ \\
\hline $2 p^{2} S$ & $1.32[-13]$ & $2 p^{2} s$ & $4.20[-14]$ & $2 s 2 p^{33} D^{\circ}$ & $1.16[-14]$ \\
\hline \multirow[t]{4}{*}{$2 p 3 d^{3} D^{\circ}$} & $9.16[-14]$ & $2 p 3 d^{3} D^{o}$ & $2.73[-14]$ & $2 p 3 d{ }^{3} F^{o}$ & $1.05[-14]$ \\
\hline & & $2 p 4 d^{3} F^{o}$ & $2.55[-14]$ & $2 p 4 d{ }^{3} F^{o}$ & $6.12[-15]$ \\
\hline & & & & $2 p 3 p{ }^{3} D$ & $5.92[-15]$ \\
\hline & & & & $2 p 3 d{ }^{3} D^{o}$ & $5.77[-15]$ \\
\hline Sum $=$ & $4.67[-12]$ & & $1.52[-12]$ & & $6.10[-13]$ \\
\hline Total $=$ & $6.57[-12]$ & & $2.03[-12]$ & & $7.16[-13]$ \\
\hline Contribution $=$ & $7.10(1) \%$ & & $7.47(1) \%$ & & $8.51(1) \%$ \\
\hline
\end{tabular}

\section{$2 p^{23} P$ \\ $2 p^{2} \mathrm{D}$ \\ $2 s 2 p^{35} S^{\circ}$ \\ $2 p^{21} S$ \\ $2 p 3 d^{3} F^{0}$ \\ $2 p 3 p^{3} D$ \\ $2 p 4 d^{3} F^{o}$ \\ ${ }^{4} P 3 p{ }^{5} D^{\circ}$ \\ $2 p 3 d^{3} D^{\circ}$}

Sum $=$

Total $=$

Contribution $=$

$1.81[-11]$

$2.65[-11]$

$6.82(1) \%$
N II: $\quad N_{\text {bnd }}=231$

$\begin{array}{ll}2 p^{23} P & 2.44[-12] \\ 2 p^{2}{ }^{\mathrm{t}} D & 1.28[-12] \\ 2 s 2 p^{35} S^{\circ} & 1.12[-12] \\ 2 p^{2}{ }^{1} S & 2.55[-13] \\ 2 p 3 d^{3} F^{\circ} & 1.88[-13] \\ 2 p 3 p{ }^{3} D & 1.29[-13] \\ { }^{4} P 3 p^{5} D^{o} & 1.13[-13] \\ 2 p 4 d^{3} F^{o} & 1.13[-13] \\ 2 p 3 d^{3} D^{o} & 1.09[-13]\end{array}$

$5.75[-12]$

$8.35[-12]$

6.88(1)\%

O III: $\quad N_{\text {bnd }}=253$

$\begin{array}{ll}2 p^{2}{ }^{3} P & 9.05[-12] \\ 2 s 2 p^{35} S^{o} & 6.69[-12] \\ 2 p^{21} D & 6.09[-12] \\ 2 s 2 p^{31} P^{o} & 5.62[-12] \\ 2 p 3 s{ }^{1} P^{\circ} & 2.81[-12] \\ 2 s 2 p^{33} D^{\circ} & 1.81[-12] \\ 2 p^{21} S & 1.71[-12] \\ { }^{4} P 3 d^{5} F & 1.46[-12] \\ { }^{4} P 3 p{ }^{5} D^{o} & 1.18[-12] \\ 2 p 3 p{ }^{3} D & 1.14[-12] \\ & \\ \text { Sum }= & 3.76[-11] \\ \text { Total }= & 6.38[-11] \\ \text { Contribution }= & 5.89(1) \%\end{array}$

$\begin{array}{ll}2 p^{23} P & 5.04[-12] \\ 2 s 2 p^{31} P^{\circ} & 2.23[-12] \\ 2 s 2 p^{35} S^{\circ} & 2.12[-12] \\ 2 p^{21} D & 1.95[-12] \\ 2 p 3 s^{1} P^{o} & 1.24[-12] \\ 2 s 2 p^{33} D^{\circ} & 6.53[-13] \\ 2 p^{2}{ }^{1} S & 5.38[-13] \\ { }^{4} P 3 d^{5} F & 4.60[-13] \\ { }^{4} P 3 p^{5} D^{\circ} & 3.77[-13] \\ 2 p 3 p^{3} D & 3.69[-13] \\ 2 p 3 p{ }^{3} S & 3.32[-13] \\ & 1.53[-11] \\ & 2.37[-11] \\ & 6.46(1) \%\end{array}$$$
2 p^{23} \mathrm{P}
$$

$\begin{array}{ll}2 p^{23} P & 8.32[-13] \\ 2 p^{2}{ }^{1} D & 4.21[-13] \\ 2 s 2 p^{35} S^{o} & 3.59[-13] \\ 2 s 2 p^{3} D^{\circ} & 3.27[-13] \\ 2 s 2 p^{3} P^{\circ} & 1.54[-13] \\ 2 p^{21} S & 8.26[-14] \\ 2 p 3 s{ }^{3} P^{\circ} & 6.17[-14] \\ 2 p 3 d{ }^{3} F^{o} & 5.26[-14] \\ 2 p 3 d{ }^{3} D & 4.89[-14] \\ { }^{4} P 3 p^{5} D^{\circ} & 3.87[-14] \\ 2 p 4 d^{3} F^{o} & 3.26[-14] \\ 2 p 3 p{ }^{3} P & 2.98[-14] \\ 2 p 3 d{ }^{3} D^{o} & 2.97[-14] \\ 2 s 2 p^{3} S^{o} & 2.24[-14] \\ & 2.49[-12] \\ & 3.15[-12] \\ & 7.91(1) \%\end{array}$

$\begin{array}{ll}2 s 2 p^{3}{ }^{3} D^{o} & 4.15[-12] \\ 2 p^{23} P & 1.62[-12] \\ 2 s 2 p^{33} P^{o} & 1.20[-13] \\ 2 s 2 p^{3} S^{o} & 6.79[-13] \\ 2 p^{21} D & 6.52[-13] \\ 2 p 3 s{ }^{3} P^{o} & 6.00[-13] \\ 2 s 2 p^{31} D^{o} & 4.41[-13] \\ 2 p 3 d^{3} F^{o} & 2.78[-13] \\ 2 s 2 p^{3}{ }^{3} P^{o} & 2.54[-13] \\ & \\ & \\ & 9.88[-12] \\ & 1.38[-11] \\ & 7.14(1) \%\end{array}$


TABLE IV. (Continued).

\begin{tabular}{|c|c|c|c|c|c|}
\hline \multicolumn{2}{|c|}{$T=100 \mathrm{~K}$} & \multicolumn{2}{|c|}{$T=1000 \mathrm{~K}$} & \multicolumn{2}{|c|}{$T=10000 \mathrm{~K}$} \\
\hline State & Coefficient & State & Coefficient & State & Coefficient \\
\hline \multicolumn{6}{|c|}{ F IV: $\quad N_{\text {bnd }}=291$} \\
\hline $2 p^{23} P$ & $2.06[-11]$ & $2 s 2 p^{33} D^{0}$ & $7.07[-12]$ & $2 s 2 p^{33} D^{\circ}$ & $4.41[-12]$ \\
\hline $2 s 2 p^{3} D^{\circ}$ & $1.50[-11]$ & $2 p^{23} P$ & $6.53[-12]$ & $2 p^{23} P$ & $2.71[-12]$ \\
\hline $2 s 2 p^{41} P^{\circ}$ & $1.38[-11]$ & $2 p 3 d^{3} F^{o}$ & $4.38[-12]$ & $2 p 3 d^{3} F^{o}$ & $2.56[-12]$ \\
\hline $2 p 3 d^{3} F^{o}$ & $1.19[-11]$ & $2 s 2 p^{31} P^{o}$ & $3.43[-12]$ & $2 s 2 p^{31} D^{o}$ & $2.29[-12]$ \\
\hline $2 p^{21} D$ & $1.08[-11]$ & $2 p^{21} D$ & $3.42[-12]$ & $2 s 2 p^{33} S^{o}$ & $2.21[-12]$ \\
\hline $2 s 2 p^{35} S^{0}$ & $8.95[-12]$ & $2 s 2 p^{35} S^{o}$ & $2.83[-12]$ & $2 s 2 p^{33} P^{o}$ & $1.78[-12]$ \\
\hline $2 s 2 p^{31} D^{o}$ & $6.14[-12]$ & $2 s 2 p^{33} P^{o}$ & $2.76[-12]$ & $2 s 2 p^{31} P^{o}$ & $1.58[-12]$ \\
\hline $2 s 2 p^{33} P^{o}$ & $5.56[-12]$ & $2 s 2 p^{31} D^{0}$ & $2.10[-12]$ & $2 p 3 d^{3} D^{\circ}$ & $1.43[-12]$ \\
\hline $2 p 3 d^{1} D^{o}$ & $3.62[-12]$ & ${ }^{4} P 3 d{ }^{3} F$ & $9.08[-13]$ & $2 p^{21} D$ & $1.27[-12]$ \\
\hline \multirow[t]{8}{*}{$2 p 3 d^{1} P^{o}$} & $3.17[-12]$ & $2 p 3 d{ }^{3} D^{o}$ & $9.00[-13]$ & $2 s 2 p^{35} S^{0}$ & $8.97[-13]$ \\
\hline & & $2 p 3 d{ }^{1} D^{o}$ & $8.89[-13]$ & & \\
\hline & & ${ }^{4} P 3 d{ }^{5} \mathrm{D}$ & $7.72[-13]$ & & \\
\hline & & $2 p 3 d^{1} P^{\circ}$ & $7.69[-13]$ & & \\
\hline & & ${ }^{4} P 3 p{ }^{3} D^{\circ}$ & $7.64[-13]$ & & \\
\hline & & $2 p 3 d^{1} F^{o}$ & $7.23[-13]$ & & \\
\hline & & $2 p^{2} s$ & $6.71[-13]$ & & \\
\hline & & $2 p 3 d^{3} D$ & $6.67[-13]$ & & \\
\hline Sum $=$ & $9.95[-11]$ & & $3.96[-11]$ & & $2.11[-11]$ \\
\hline Total $=$ & $1.64[-10]$ & & $5.59[-11]$ & & $2.97[-11]$ \\
\hline \multirow[t]{2}{*}{ Contribution $=$} & $6.06(1) \%$ & & $7.09(1) \%$ & & $7.12(1) \%$ \\
\hline & & \multicolumn{2}{|c|}{ Ne v: $\quad N_{\text {bnd }}=321$} & & \\
\hline $2 s 2 p^{33} D^{0}$ & $1.39[-10]$ & $2 s 2 p^{33} D^{o}$ & $6.81[-11]$ & $2 s 2 p^{3} D^{o}$ & $1.47[-11]$ \\
\hline $2 p^{23} P$ & $2.66[--11]$ & $2 p^{43} P$ & $3.40[-11]$ & $2 s 2 p^{33} P^{o}$ & $1.27[-11]$ \\
\hline $2 s 2 p^{3} P^{o}$ & $1.28[-11]$ & $2 s 2 p^{3}{ }^{3} P^{\circ}$ & $3.38[-11]$ & $2 p^{43} P$ & $6.53[-12]$ \\
\hline $2 p^{21} D$ & $1.23[-11]$ & $2 p^{23} P$ & $8.59[-12]$ & $2 p^{21} D$ & $4.97[-12]$ \\
\hline $2 s 2 p^{35} S^{o}$ & $1.21[-11]$ & ${ }^{2} D 3 s^{3} D$ & $8.52[-12]$ & ${ }^{2} D 3 d{ }^{3} G$ & $4.76[-12]$ \\
\hline${ }^{2} D p^{3} D^{\circ}$ & $1.00[-11]$ & ${ }^{2} P 3 s^{3} P$ & $7.13[-12]$ & ${ }^{2} D 3 d{ }^{3} F$ & $4.71[-12]$ \\
\hline $2 p 4 d^{3} D^{\circ}$ & $9.39[-12]$ & $2 p^{21} D$ & $3.94[-12]$ & $2 p^{23} P$ & $3.49[-12]$ \\
\hline $2 p 3 d^{3} F^{o}$ & $8.19[-12]$ & $2 s 2 p^{35} S^{0}$ & $3.82[-12]$ & ${ }^{2} D 3 d^{3} D$ & $2.85[-12]$ \\
\hline $2 p 4 d^{3} F^{o}$ & $7.82[-12]$ & ${ }^{2} D 3 p{ }^{3} D^{\circ}$ & $3.64[-12]$ & $2 s 2 p^{31} P^{o}$ & $1.63[-12]$ \\
\hline${ }^{2} D 3 p{ }^{3} F^{\circ}$ & $7.62[-12]$ & $2 s 2 p^{31} D^{0}$ & $3.60[-12]$ & ${ }^{2} D 3 d{ }^{1} F$ & $1.48[-12]$ \\
\hline \multirow[t]{7}{*}{$2 p 3 d^{3} D^{o}$} & $6.14[-12]$ & ${ }^{2} D 3 p{ }^{1} F^{\circ}$ & $3.50[-12]$ & ${ }^{2} D 3 d^{1} G$ & $1.45[-12]$ \\
\hline & & ${ }^{2} D 3 p{ }^{3} P^{o}$ & $3.17[-12]$ & $2 p^{21} D$ & $1.29[-12]$ \\
\hline & & $3 p 4 d^{3} D^{\circ}$ & $3.08[-12]$ & $2 s 2 p^{35} S^{0}$ & $1.27[-12]$ \\
\hline & & & & ${ }^{2} D 3 s{ }^{3} D$ & $1.27[-12]$ \\
\hline & & & & ${ }^{2} D 3 p{ }^{3} P^{o}$ & $1.20[-12]$ \\
\hline & & & & ${ }^{2} P 3 s^{3} P$ & $1.04[-12]$ \\
\hline & & & & ${ }^{2} D 3 p^{1} F^{o}$ & $1.04[-12]$ \\
\hline Sum $=$ & $2.52[-10]$ & & $1.85[-10]$ & & $6.64[-11]$ \\
\hline Total $=$ & $3.56[-10]$ & & $2.32[-10]$ & & $8.78[-11]$ \\
\hline \multirow[t]{2}{*}{ Contribution $=$} & $7.09(1) \%$ & & $7.85(1) \%$ & & $7.57(1) \%$ \\
\hline & & \multicolumn{2}{|c|}{$N_{\text {bnd }}=469$} & & \\
\hline $2 p^{23} p$ & $1.66[-10]$ & $2 p^{43} P$ & $1.05[-9]$ & $2 p^{43} P$ & $1.24[-10]$ \\
\hline $2 p^{43} P$ & $8.15[-11]$ & $2 p^{23} P$ & $1.66[-10]$ & $2 s 2 p^{33} P^{o}$ & $3.85[-11]$ \\
\hline $2 p^{2 \mathrm{I}} D$ & $3.29[-11]$ & ${ }^{2} D^{\circ} 3 p{ }^{3} D$ & $1.48[-10]$ & $2 p^{41} D$ & $3.01[-11]$ \\
\hline $2 s 2 p^{33} P^{o}$ & $3.10[-11]$ & ${ }^{2} D^{\circ} 3 p{ }^{3} P$ & $8.53[-11]$ & $2 p^{23} P$ & $1.93[-11]$ \\
\hline $2 s 2 p^{35} S^{0}$ & $2.25[-11]$ & $2 s 2 p^{33} P^{0}$ & $6.84[-11]$ & ${ }^{2} D^{\circ} 3 d^{3} G^{o}$ & $1.84[-11]$ \\
\hline${ }^{2} P^{o} 3 p{ }^{3} D$ & $2.20[-11]$ & ${ }^{2} P 3 s{ }^{3} P$ & $3.85[-11]$ & ${ }^{2} D^{o} 3 d^{3} F^{\circ}$ & $1.48[-11]$ \\
\hline${ }^{2} P 3 s{ }^{3} P$ & $1.85[-11]$ & $2 p^{41} D$ & $3.37[-11]$ & ${ }^{2} D^{o} 3 p^{3} D$ & $1.19[-11]$ \\
\hline $2 p 5 p^{3} D$ & $1.70[-11]$ & ${ }^{2} P^{0} 3 p{ }^{3} D$ & $2.67[-11]$ & ${ }^{2} D^{\circ} 3 p^{3} P$ & $9.70[-12]$ \\
\hline${ }^{2} P^{o} 3 p{ }^{3} P$ & $1.66[-11]$ & $2 p 5 p^{3} D$ & $2.43[-11]$ & ${ }^{2} S 3 p^{3} P^{\circ}$ & $8.31[-12]$ \\
\hline${ }^{2} P 4 s{ }^{3} P$ & $1.52[-11]$ & & & $2 p^{21} D$ & $7.59[-12]$ \\
\hline
\end{tabular}


TABLE IV. (Continued).

\begin{tabular}{|c|c|c|c|c|c|}
\hline \multicolumn{2}{|c|}{$T=100 \mathrm{~K}$} & \multicolumn{2}{|c|}{$T=1000 \mathbf{K}$} & \multicolumn{2}{|c|}{$T=10000 \mathrm{~K}$} \\
\hline State & Coefficient & State & Coefficient & State & Coefficient \\
\hline $2 p 3 d^{3} F^{o}$ & $1.27[-11]$ & & & ${ }^{2} D^{\circ} 3 d^{1} G^{o}$ & $5.25[-12]$ \\
\hline${ }^{2} D^{\circ} 3 p^{3} D$ & $1.16[-11]$ & & & ${ }^{2} S 3 d d^{3} D$ & $5.06[-12]$ \\
\hline${ }^{4} P 3 d^{5} F$ & $1.13[-11]$ & & & $2 s 2 p^{31} P^{o}$ & $4.70[-12]$ \\
\hline $2 p 3 d^{3} D$ & $1.10[-11]$ & & & & \\
\hline Sum $=$ & $4.70[-10]$ & & $1.65[-9]$ & & $2.98[-10]$ \\
\hline Total $=$ & $8.84[-10]$ & & $1.99[-9]$ & & $4.20[-10]$ \\
\hline Contribution $=$ & $5.32(1) \%$ & & $8.26(1) \%$ & & $7.09(1) \%$ \\
\hline
\end{tabular}

C II: $\quad N_{\text {bnd }}=67$

$\begin{array}{llllll}2 s^{2} 2 p^{2} P^{o} & 1.13[-11] & 2 s^{2} 2 p^{2} P^{o} & 3.61[-12] & 2 s^{2} 2 p^{2} P^{o} & 1.23[-12] \\ 2 s 2 p^{24} P & 5.83[-12] & 2 s 2 p^{2}{ }^{4} P & 1.84[-12] & 2 s 2 p^{2}{ }^{2} D & 1.17[-12] \\ 2 s^{2} 3 d^{2} D & 1.36[-12] & 2 s 2 p^{2} D & 5.66[-13] & 2 s 2 p^{24} P & 5.81[-13] \\ 2 s^{2} 3 p^{2} P^{o} & 1.29[-12] & 2 s^{2} 3 d^{2} D & 4.14[-13] & 2 s^{2} 3 p^{2} P^{o} & 1.41[-13] \\ 2 s 2 p^{2} D & 9.86[-13] & 2 s^{2} 3 p^{2} P^{o} & 4.10[-13] & 2 s^{2} 3 d^{2} D & 1.11[-13] \\ 2 s^{2} 4 d^{2} D & 8.46[-13] & 2 s^{2} 4 d^{2} D & 2.61[-13] & 2 s 2 p 3 s^{4} P^{o} & 9.60[-14] \\ 2 s 2 p 3 d^{4} F^{o} & 6.48[-13] & 2 s 2 p 3 d^{4} F^{o} & 2.03[-13] & & \\ 2 s^{2} 4 f^{2} F^{\circ} & 5.67[-13] & 2 s 2 p 3 p^{4} D & 1.77[-13] & \\ 2 s 2 p 3 p^{4} D & 5.58[-13] & 2 s^{2} 4 f^{2} F^{o} & 1.73[-13] & \\ 2 s^{2} 5 d^{2} D & 5.24[-13] & 2 s^{2} 5 d^{2} D & 1.63[-13] & \\ 2 s^{2} 5 f^{2} F^{o} & 5.11[-13] & 2 s^{2} 5 f^{2} F^{o} & 1.56[-13] & \\ 2 s^{2} 6 f^{2} F^{o} & 3.81[-13] & 2 s^{2} 4 p^{2} P^{o} & 1.17[-13] & \\ 2 s^{2} p 3 p^{4} P & 3.51[-13] & & & \\ 2 s^{2} 6 d^{2} D & 3.32[-13] & & & \\ 2 s^{2} 6 g^{2} G & 3.03[-13] & & & & \\ \text { Sum }= & 2.62[-11] & & 8.09[-12] & \\ \text { Total= } & 3.71[-11] & & 1.15[-11] & \\ \text { Contribution= } & 7.06(1) \% & & 7.04(1) \% & \end{array}$

S II: $\quad N_{\text {bnd }}=315$

\begin{tabular}{|c|c|c|c|c|c|}
\hline${ }^{5} S^{\circ} 3 d^{6} D^{\circ}$ & $4.75[-12]$ & $3 p^{32} D^{o}$ & $1.90[-12]$ & $3 p^{32} D^{o}$ & $5.79[-13]$ \\
\hline $3 p^{32} P^{o}$ & $2.45[-12]$ & ${ }^{5} S^{o} 3 d^{6} D^{0}$ & $1.50[-12]$ & ${ }^{5} S^{\circ} 3 d^{6} D^{\circ}$ & $4.63[-13]$ \\
\hline $3 p^{2} 3 d^{4} F$ & $1.72[-12]$ & $3 p^{32} P^{o}$ & $1.02[-12]$ & $3 p^{32} P^{o}$ & $3.90[-13]$ \\
\hline $3 p^{32} D^{\circ}$ & $1.67[-12]$ & $3 p^{2} 3 d^{4} F$ & $5.06[-13]$ & $3 s 3 p^{44} P$ & $3.57[-13]$ \\
\hline $3 p^{34} S^{0}$ & $1.15[-12]$ & $3 p^{2} 3 d^{4} D$ & $4.60[-13]$ & $3 p^{2} 3 d^{4} F$ & $2.10[-13]$ \\
\hline $3 p^{2} 3 d^{4} D$ & $8.51[-13]$ & $3 p^{34} S^{o}$ & $3.23[-13]$ & $3 p^{2} 3 d^{4} D$ & $1.39[-13]$ \\
\hline $3 p^{2} 3 d^{2} P$ & $6.26[-13]$ & $3 s 3 p^{44} P$ & $1.98[-13]$ & $3 s 3 p^{42} D$ & $1.37[-13]$ \\
\hline $3 s 3 p^{44} P$ & $4.57[-13]$ & $3 p^{2} 3 d^{2} P$ & $1.42[-13]$ & $3 p^{34} S^{o}$ & $1.32[-13]$ \\
\hline $3 p^{2} 3 d^{2} F$ & $3.73[-13]$ & $3 p^{2} 3 d^{2} F$ & $1.39[-13]$ & $3 p^{2} 3 d^{2} F$ & $8.58[-14]$ \\
\hline${ }^{5} S^{\circ} 4 p{ }^{6} P$ & $3.68[-13]$ & ${ }^{5} S^{0} 4 p^{6} P$ & $1.15[-13]$ & $3 p^{2} 3 d^{4} P$ & $8.49[-14]$ \\
\hline $3 s 3 p^{42} D$ & $3.08[-13]$ & $3 p^{2} 4 d^{4} F$ & $9.73[-14]$ & $3 p^{2} 3 d^{2} P$ & $5.61[-14]$ \\
\hline $3 p^{2} 4 d^{4} F$ & $2.96[-13]$ & $3 s 3 p^{42} D$ & $7.18[-14]$ & ${ }^{1} D 3 d^{2} G$ & $4.46[-14]$ \\
\hline $3 p^{2} 4 d^{4} D$ & $2.15[-13]$ & $3 p^{2} 3 d^{4} P$ & $6.46[-14]$ & & \\
\hline $3 p^{2} 3 d^{4} P$ & $2.09[-13]$ & $3 p^{2} 4 f^{4} G^{o}$ & $6.39[-14]$ & & \\
\hline \multirow[t]{2}{*}{$3 p^{2} 4 f^{4} G^{o}$} & $2.09[-13]$ & $3 p^{2} 5 d^{4} D$ & $6.12[-14]$ & & \\
\hline & & ${ }^{1} D 4 p^{2} D^{\circ}$ & $5.91[-14]$ & & \\
\hline Sum $=$ & $1.57[-11]$ & & $6.72[-12]$ & & $2.68[-12]$ \\
\hline Total $=$ & $2.43[-11]$ & & $9.52[-12]$ & & $3.76[-12]$ \\
\hline Contribution $=$ & $6.44[1] \%$ & & $7.06[1] \%$ & & $7.12[1] \%$ \\
\hline
\end{tabular}


Problems may arise in averaging over the resonances for ions with closely spaced excited thresholds, and their QDT regions overlap. (An example is target $\mathbf{S}$ III in Table I where two excited states, $3 s^{2} 3 p 3 d^{3} P^{o}$ and $3 s^{2} 3 p 4 s^{3} P^{o}$, have overlapping QDT regions.) For both the low- $n$ and the high- $n$ states' calculations, we may treat these states as degenerate. This will be discussed more later.

The calculations for specific atomic systems are described below. Although all parts of the calculations have been carried out in their entirety for the present work, most of the details of the atomic structure and the photoionization calculations have been omitted since these are essentially the same as for other OP calculations.

The eigenfunction expansion for the target ions in the boron sequence is taken to be as in the earlier work by Luo and Pradhan [19] and NP1. In the latter work, photoionization from, and low- $n$ recombination into, are described for a few C-like ions. The present work extends the NP1 calculations by the inclusion of DR (as outlined briefly in NP2), and includes recombination into other ions in the $\mathrm{C}$ sequence. The atomic structure calculations and the relevant data for the target B-like ions, the computed energy levels and oscillator strengths, are described by Luo and Pradhan [19] in their calculations for the OP. In the present work, although we use the same target configurations, we employ observed energies following the diagonalization of the $e+$ ion Hamiltonian; a procedure which ensures the precise positions of the target thresholds to which the autoionizing resonances converge (for some ions a complete set of spectroscopic energies is not available and the calculated target energies are used). Table I gives the atomic structure data and the configuration-interaction (CI) expansions for the eight target terms included for the B-like ions: $2 s^{2} 2 p\left({ }^{2} P^{0}\right)$, $2 s 2 p^{2}\left({ }^{4} P,{ }^{2} D,{ }^{2} S,{ }^{2} P\right), 2 p^{3}\left({ }^{4} S^{o},{ }^{2} D^{o},{ }^{2} P^{o}\right)$. For C II we also include $2 s^{2} 3 s\left({ }^{2} S\right)$ and $2 s^{2} 3 p\left({ }^{2} P^{o}\right)$, since these terms lie in between the terms dominated by the $2 p^{3}$ configuration.

Photoionization calculations are carried out for all bound states of the C-like ions with $n_{0}=10$ and $l \leq l_{\max }=5$. As it is important to resolve the often narrow resonances close to the ionization threshold (responsible for "low-temperature DR" as discussed by Nussbaumer and Storey [2],) we divide the photoionization calculations into two energy ranges: (i) up to $0.2 \mathrm{Ry}$ above the ionization threshold where we employ a fine mesh of 500 energies, and (ii) an effective quantum number mesh, for energies above $0.2 \mathrm{Ry}$, with $\Delta v=0.01$. The mesh in (ii) ensures 100 points in between each interval $(\nu, \nu+1)$. In NP1 we have described some of these calculations in detail, including a list of the large number of bound states included for CI, N II, and O III. For brevity we do not list all the states of each atom or ion in this report, but the total number of states considered, $N_{\text {bnd }}$, is given in the later discussion on individual contributions of particular bound states (Table IV).

The DR calculations for states with $n_{0}<n \leq \infty$ are essentially for electron-ion scattering. The partial waves included for electron scattering with the target B-like ions are $l \leq 9$. Coupled with the target states given above the total $S L \pi$ states are given by the multiplicities
$(2 S+1)=1,3$, and 5 , and $L=0-10$.

Although the two sets of results for Maxwellianaveraged DR rate coefficients using the detailed and the averaged collision strengths agree to within a few percent, the values obtained from the analytical averaging procedure are presented. However, there may be cases where the analytic averaging procedure may not be reliable owing to overlapping QDT regions of successive thresholds and the DR collision strengths would need to be obtained from the detailed collision strengths. As the resonances in the small QDT region are very narrow, and may have overlapping structures due to interference between the several Rydberg series of resonances, it is necessary to use a very fine mesh in energy, particularly just below threshold where the DR collision strength rises rapidly.

The 17-state eigenfunction expansion and related atomic data for the target ion S III are given in Table I, and the associated radiative transition rates for the relevant core transitions in Table II. The CC calculations for photoionization cross sections are carried out for $N_{\text {bnd }}=315$ bound states with $n_{0}=10$ and $l_{\max }=9$. These photoionization cross sections are an improvement over the OP cross sections with a bigger eigenfunction expansion. Photoionization cross sections of a few states of S II are presented in Fig. 3. $\Omega(\mathrm{DR})$ for $\mathrm{S}$ II are calculated with partial waves up to $l_{\max }=9$ and are presented in Figs. 4 and 5 (results are described in the next section).

The C III target ion is represented by a 12-state eigenfunction expansion (Table I), also a bigger expansion considered in the OP. In other respects the close-coupling calculations are similar to the other ions with $n_{0}=10$ and $l_{\max }=9$. The number of bound states of C II involved in the photoionization calculations is 67 .

Some general features of the computations for the low- $n$ and the high- $n$ parts are described in the next two sections.

\section{A. Dominant recombinations to low- $n$ states}

Out of the several hundred bound states of the $e+$ ion system considered in the low- $n$ region of recombination, it is found that a relatively smaller number may make the dominant contribution. Table IV gives a list of these bound states, their individual contributions, and the cumulative percentage of the listed states to the total rate coefficient. The contributions of the dominant states are listed at three temperatures: $10^{2}, 10^{3}$, and $10^{4} \mathrm{~K}$. Owing to the extensive resonance structures in photoionization cross sections there can be considerable variation in the magnitude of recombination through a given bound state with electron temperature. The states included in Table IV are such that their contributions are comparable. At low temperatures, the ground-state recombination usually (but not always - as we show later) dominates the total. As the temperature increases, recombination through other excited states becomes more prominent; the number of states can be quite large at some temperatures, as also seen from Table IV.

Particularly large contributions may come from excited metastable states when their effective photoionization 
cross sections are enhanced significantly by autoionization. For example, the $2 p^{4}\left({ }^{3} P\right)$ metastable state of Si IX makes a considerable contribution to recombination rate at low temperatures as seen in Table IV, since its photoionization cross section at low energies is dominated by autoionizing resonances (the central subplot in Fig. 2). Below the first ionization threshold for the $2 p$ optical electron, ${ }^{4} S^{\circ}$, i.e.,

$$
2 p^{4}\left({ }^{3} P\right)+h v \rightarrow 2 p^{3}\left({ }^{4} S^{o}\right)+e,
$$

the photoionization proceeds only through autoionization in the fairly large energy region between the ground state of the residual ion $2 p\left({ }^{2} P^{\circ}\right)$ and the $2 p^{3}\left({ }^{4} S^{\circ}\right)$ state, from about 19.5 to about $24 \mathrm{Ry}$ in Fig. 2. (See also NP1.)

\section{B. Electron impact excitation and dielectronic recombination}

The generalized unitarity condition for the electron and the photon flux relates the electron-impact-excitation and DR collision strengths, i.e., the EIE with allowance for radiation damping of electrons results in the photon flux from DR. In particular, we calculate EIE collision strengths $\Omega$ (EIE) for the dipole transitions from the ground state of the target ions and compare with the peak, resonance-averaged DR collision strengths

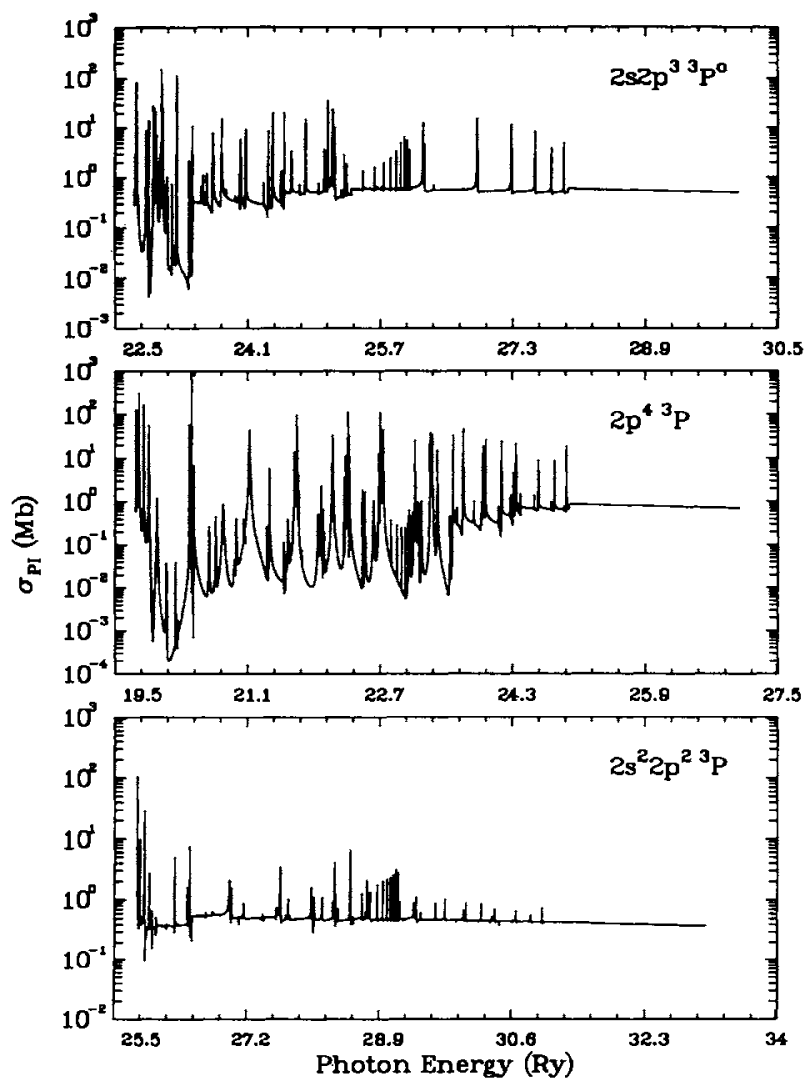

FIG. 2. Photoionization cross sections for the three states of Si IX that make a dominant contribution to the low- $n$ and lowtemperature recombination rate coefficients.
( $\Omega(D R)\rangle$ at the corresponding thresholds. This provides an important consistency check on the DR calculations. The DR collision strengths are calculated in what we call the QDT region where the $\chi$ matrices are obtained from the asymptotic continuum wave functions, outside the $R$-matrix boundary $r=a$, neglecting the long-range multipole (non-Coulomb) potentials. In the outer region, $r>a$, the coupled integro-differential equations of the close-coupling theory reduce to coupled differential equations (DE) [7]

$$
\left(\frac{d^{2}}{d r^{2}}-\frac{l(l+1)}{r^{2}}+\frac{2 z}{r}+\varepsilon\right) F(r)=\sum_{i^{\prime}} V_{i i^{\prime}} F_{i^{\prime}}(r),
$$

where the $V_{i i^{\prime}}(r)$ are long-range multipole potentials

$$
V_{i i^{\prime}}(r)=\sum_{\lambda} C_{i i^{\prime}}^{\lambda} / r^{\lambda+1}
$$

In actual $R$-matrix calculations the contributions from $\lambda=1$ and 2 are included. The target functions $\psi_{i}$ in Eq. (1) are small for $r \geq a$, and it follows that [20]

$$
\left|V_{i i}(r)\right| \ll \frac{2 z}{r} \quad(r \geq a)
$$

The multipole potentials may therefore be treated as small perturbations and in the calculation of the $\chi$ matrix elements in the QDT regions where we have Rydberg resonances in high- $n$ states, are neglected in the DR calculations. The solutions of the DE's are then Coulomb functions [21]. Whether or not this approximation is valid is indicated by the discrepancy between the DR collision strengths at threshold (peak values) and the EIE collision strengths calculated including the long-range multipole potentials. We calculate the $\Omega$ (EIE), at the thresholds in question, in three different approximations that are characterized in the asymptotic-region code by the parameter IPERT: (i) neglecting the long-range potentials $V_{i i^{\prime}}($ IPERT $=0)$, (ii) including the $V_{i i^{\prime}}($ IPERT $=1)$, and (iii) including $V_{i i^{\prime}}$ and completing the partial-wave summation in $l, l_{\max }<l \leq \infty$, for the dipole-allowed transitions using the Coulomb-Bethe-type approximation (IPERT $=2)$. The condition (iii) is important in that we need to ensure that all partial waves contributing to electron-ion scattering have been included in the EIE and the DR calculations. In the current work we consider $l \leq l_{\max }=9$ for all ions and find close agreement between the IPERT $=2$ collision strengths and the other two cases, indicating that the included partial waves are sufficient.

Usually one chooses the $R$-matrix boundary with the criterion that the bound target orbitals $P_{n l}(r)$ have decayed exponentially to sufficiently small values at $r=a$, and that the outer electron is predominantly under the influence of the Coulomb potential for $r>a$. However, this criterion does not always ensure that the effect of multipole potentials is negligible for all transitions between the target states. In case of discrepancy between the IPERT $=0$ and the IPERT $=1$ values therefore, we increase the $R$-matrix boundary $(R A)$ so that the effect of the multipole potentials is confined to the inner region and a reasonable agreement is obtained between the two 
sets of collision strengths. This also implies that the DR collision strengths are calculated using the $\chi$ matrices that pertain to the asymptotic region, where the Coulomb potential is dominant. In practice, it is sometimes also necessary to increment the number of continuum basis orbitals in the $R$-matrix basis set for the enlarged $R A$. A number of trials are thus necessary. For certain ions, the entire set of $R$-matrix calculations is repeated several times to achieve consistent results between EIE and DR at the target thresholds of interest.

Table III gives the five sets of data for collision strengths for each of the ions reported in this work: the three values from the IPERT $=0,1$, and 2 calculations at the target thresholds, the resonance-averaged DR collision strength $\langle\Omega(D R)\rangle$, and the actual $D R$ collision strength $\Omega(\mathrm{DR})$ at threshold. The generally good agreement, of the order of a few percent, between the five sets of collision strengths at each threshold is indicative of the fact that both the multipole potentials and the partialwave summation have been correctly accounted for. For example, for the Ne VI target, we have chosen $R A=6.4$ bohrs radii, which results in no more than about $10 \%$ discrepancy between the IPERT $=0$ and 1 threshold EIE collision strengths for the three dipole transitions ${ }^{2} P^{o} \rightarrow{ }^{2} D,{ }^{2} S,{ }^{2} P$. However, with $R A=3$. 1 we found that the collision strengths differ by up to $50 \%$ (although the bound orbitals still decay down to about 0.001 ). The maximum discrepancy was for the first transition ${ }^{2} P^{o} \rightarrow{ }^{2} D$, for which the IPERT $=0$ and 1 values, with smaller $R A=3.1$, are 2.4 and 3.6, respectively.

The DR rate coefficients for the high- $n$ states in the present work are obtained from the analytic expression for $\langle\Omega(D R)\rangle$ which is numerically stable. However, there may be overlapping QDT regions of target states that are related to the ground state via dipole transitions. An example is the Ne VI target ion where the QDT regions of the ${ }^{2} S$ and the ${ }^{2} P$ thresholds overlap to some extent. Consequently the excitation collision strength at the ${ }^{2} S$ threshold differs from $\langle\Omega(D R)\rangle$ by about a factor of 2 (Table III), owing to the presence of strongly closed ${ }^{2} P$ channels. Normally one can obviate the problem by setting the thresholds degenerate, which is seen to alter the DR rate coefficient by only a few percent. Therefore we do not expect any significant error in the DR results for Nev.

Even though it is difficult to obtain a fine enough energy resolution to compute the detailed profiles of the DR collision strengths, particularly near the peak value at thresholds, it is of interest to note from Table III that the $\langle\Omega(D R)\rangle$ in general agrees well within $10 \%$ with the actual numerical value of the detailed $\Omega(D R)$ at the threshold. This is of potential importance in cases where the averaged DR values may not be reliable owing to overlapping QDT regions and the presence of resonances from strongly closed channels within the region of the narrow resonances from the weakly closed channels as in the case of $\mathrm{NeV}$ discussed above. In such cases the DR contributions may be obtained from the detailed $\Omega(D R)$. Although the results in the present work are largely unaffected by this problem, we are studying cases where the detailed $\Omega(D R)$ itself may be needed and then it would be necessary to employ extremely fine energy resolution.

\section{RESULTS AND DISCUSSION}

Total electron-ion recombination cross sections and rate coefficients have been calculated for a number of atomic systems. In the present report, however, we present sample results only for the ions described in the preceding section. The numerical values for the rate coefficients will be presented elsewhere. As may be inferred, the calculations involve a huge amount of data in the intermediate stages: photoionization cross sections for typically a few hundred bound states, at a few thousand energies for each cross section to delineate the resonances in the photoionization of each state; the contribution to recombination rate coefficient from each bound state in the low- $n$ region; the detailed and resonance-averaged DR collision strengths and EIE collision strengths for a number of partial waves and symmetries; hydrogenic top-up for the small continuum contributions from highly excited states; the total recombination rate coefficients at an extended range of temperatures. Results presented herein pertain to the main aspects of these calculations.

\section{A. Low- $n$ recombination}

The computations are as described in the preceding section. (Some results for $\mathrm{C}$-like ions up to $\mathrm{Ne} \mathrm{v}$ have been reported previously $[4,5]$.) One of the main points that emerges, common to all atomic systems considered, is that the contribution to recombination at very low temperatures is from relatively few states of the $e+$ ion system, particularly if the low-energy autoionizing resonances are prominent. However, as the temperature increases, a number of excited states begin to contribute. For example, Fig. 2 shows the photoionization cross sections for the three states of Si IX, $2 s^{2} 2 p^{2}\left({ }^{3} P\right), 2 p^{4}\left({ }^{3} P\right)$, $2 s 2 p^{3}\left({ }^{3} P^{o}\right)$ (see also Table IV), that make the largest contributions at the three temperatures given. As discussed in the preceding section, $\sigma\left(2 p^{4}\left({ }^{3} P\right)\right)$ of $\mathrm{Si}$ IX is dominated by autoionization all throughout the low-energy region; the corresponding plot in Fig. 2 shows that these resonances increase the effective cross section by orders of magnitude.

It might be noted here that if the excited states' photoionization is treated in approximations such as the hydrogenic, the central field, or the single configuration Hartree-Fock, then this large resonant effect will be absent and the corresponding recombination rates (e.g., R R rates) would likely be in substantial error. The photoionization cross section of excited states may be highly nonhydrogenic and, as seen from Fig. 2, the "background" cross section may be inconsequential or even nonexistent. It might also be noted that $\mathrm{Si}$ IX is a fairly highly charged ion and that the effect of resonances on photoionization may not decrease with ion charge, contrary to the conclusion in some recent works [22].

The individual rate coefficients given in Table IV are also important for the formation of recombination line 
spectra wherein recombination to a specific excited state is followed by radiative cascades. Such work is in progress.

An interesting pattern of low- $n$ recombination is seen in S II (Table IV), where unexpectedly a relatively highly excited metastable state $3 s 3 p^{3}\left({ }^{5} S^{\circ}\right) 3 d\left({ }^{6} D^{o}\right)$ makes the largest contribution at low temperatures $\left(<10^{3} \mathrm{~K}\right)$. Furthermore, two lower metastable states, $3 s^{2} 3 p^{3}\left({ }^{2} D^{\circ}\right)$ and $3 s^{2} 3 p^{2} 3 d\left({ }^{4} F\right)$, the next two in terms of their magnitudes, have a higher recombination rate coefficient than the ground state $3 s^{2} 3 p^{3}\left({ }^{4} S^{o}\right)$. The photoionization cross sections for these four states are shown in Fig. 3. The extensive resonance structures are primarily responsible for the large contributions from these states, as well as the variation of their relative contributions with temperature. At very low temperatures the ${ }^{6} D^{\circ}$ has a large background cross section, whereas the others are smaller. As the temperature increases, first the ${ }^{2} D^{o}\left(\approx 10^{3}-10^{4} \mathrm{~K}\right)$ and then the ${ }^{4} F\left(\approx 10^{5} \mathrm{~K}\right)$ provide the largest single rate coefficient.

The contribution of excited states to electron-ion recombination is in general nonhydrogenic even for highly excited Rydberg states that one might expect to be represented well by methods that do not allow for the coupling of continuum photoionization channels. The reason is the presence of the photoexcitation-of-core(PEC) type resonances in the photoionization cross sec-

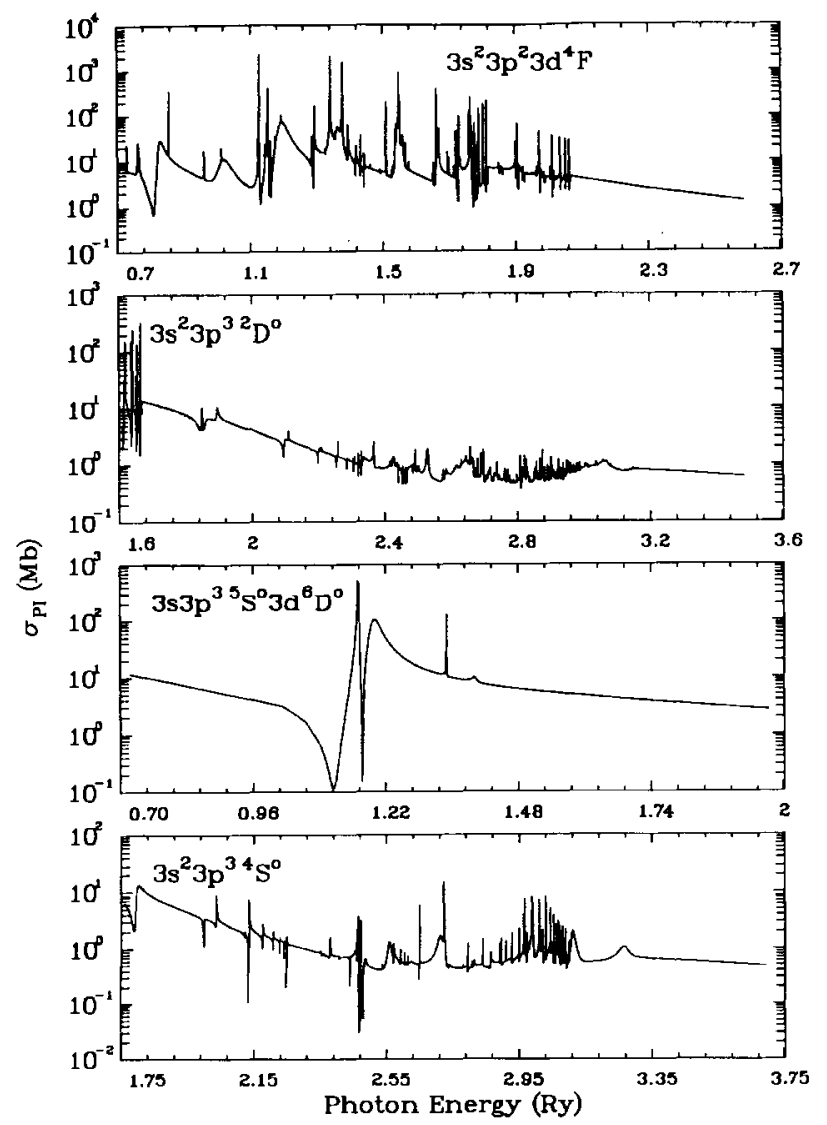

FIG. 3. Photoionization cross sections for the four states of $S$ II that make a dominant contribution to the low- $n$ and lowtemperature recombination rate coefficients. tions of Rydberg states $\left(S_{t} L_{t}\right) n l$, which show very large and broad resonances at frequencies associated with the core dipole transitions $[23,4]$. These resonances can enhance the cross section by orders of magnitude. The PEC phenomenon is akin to the DR process whereby the resonant excitation of the target core, via a strong dipole transition, plays a dominant role close to the corresponding frequencies.

Finally, the results of Table IV indicate that while the dominant, about two-thirds to three-quarters, of the low$n$ contribution at low temperatures derives from the relatively few states listed, the contribution of a large number of higher excited states is non-negligible and increases with temperature for most ions (see the cumulative percentage in Table IV). Furthermore, it is necessary to include all the bound states for accuracy as well as for completeness. The latter point is an important one in that it is essential to include all final $e+$ ion bound states to which the autoionizing states, with $n<n_{0}$, may possibly decay radiatively. If the completeness condition is not satisfied, or if the autoionization profiles, particularly in the near threshold region, are not fully resolved, then the total recombination to low- $n$ states will not be entirely accounted for. Another important point is that the recombination contribution from low- $n$ states is significant even in the high-energy and high-temperature regions where DR dominates but does not account fully for total recombination, as we shall see in the examples below.

\section{B. High-n recombination}

The predominant contribution for high- $n$ recombination is from DR (the continuum contribution, calculated in the hydrogenic approximation is found to be less than 1\%). The detailed and averaged DR collision strengths are calculated as a function of $\boldsymbol{v}$. Results for the detailed $\Omega(D R)$, including resonance structures in the emitted photon spectrum have not been reported previously, and are presented. Following is a description of the collision strengths for some of the ions.

\section{DR collision strengths for $e+S$ III $\rightarrow$ S II}

Figure 4 shows the detailed $\Omega(\mathrm{DR})$ for the few lowest sets of resonances in the high- $n(n>10)$ region. Each set belongs to a complex of autoionizing states with the $n$ value shown. The resonances belong to the Rydberg series converging on to the excited states $3 s 3 p^{3}{ }^{3} D^{o}$ which accounts for the first dipole transition from the ${ }^{3} P$ ground state of S III. The pattern of resonances in the complexes repeats itself but gets narrower with $n$, while the background rises, as judged by the minima in between the complexes. There is a considerable amount of detail within each complex reflected by the overlapping resonance profiles. The total $\Omega(D R)$ is a sum of approximately 60 ( $e+$ ion) $S L \pi$ symmetries of S II, with $l \leq 9$, many of which contribute to the DR resonance structures shown.

In contrast with the theory used in the present work, the simpler individual processes or the individual resonance approximations account for the DR contributions from these resonances with the ratio $\approx\left(A_{a} A_{r}\right) /\left(A_{a}+A_{r}\right)$, where $A_{a}$, the autoionization 


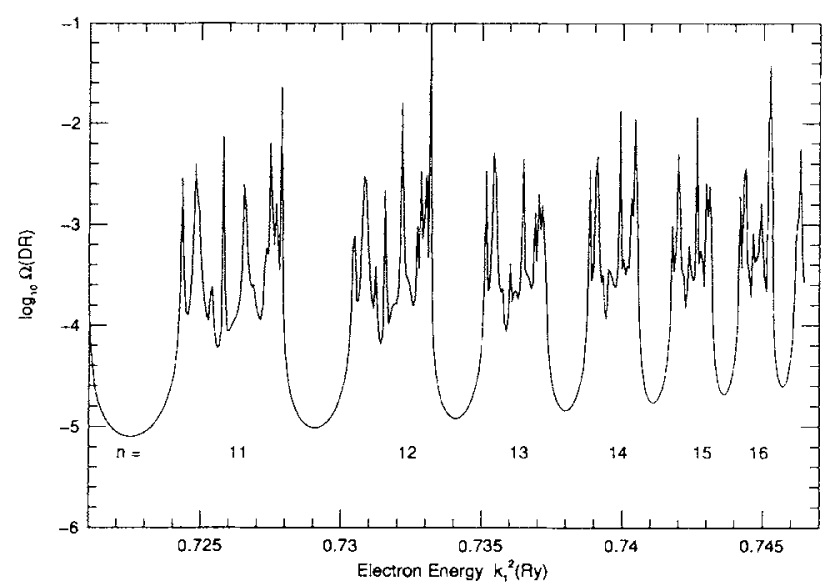

FIG. 4. Detailed DR collision strength. The resonances correspond to autoionizing states within the given $n$ complexes. The example is for $e+\mathrm{SIII} \rightarrow \mathrm{S}$ II where the resonances converge onto the excited S III state $3 s 3 p^{3}\left({ }^{3} D^{o}\right)$, and the emitted photon spectrum shown is due to radiative transition to the ground state $3 s^{2} 3 p^{2}\left({ }^{3} P\right)$.

probability, $A_{r}$, the radiative transition probability, are calculated independently. It is clear from Fig. 4 for the detailed $\Omega(D R)$ that the precise energy dependence is much more involved, even though statistical averaging over a large number of resonances might ensure that the differences in the DR rate may not be large. The effects of channel coupling and of radiation damping of resonances are manifest, and included in the present work.

Table III lists the six dipole transitions in the S III core ion. The $\Omega(D R)$ for S II, over the entire range of interest, is shown in Fig. 5. Calculations are carried out in the QDT regions $(10.0 \leq v \leq \infty)$ of these target thresholds for the detailed $\Omega(D R)$ and $\langle\Omega(D R)\rangle$. The limiting values of the averaged peaks and the convergent limits of resonances correspond to the target thresholds $3 s 3 p^{3}\left({ }^{3} D^{o},{ }^{3} P^{o},{ }^{3} S^{o}\right), \quad 3 p 3 d\left({ }^{3} P^{o}\right), \quad$ and $3 p 4 s\left({ }^{3} D^{\circ}\right)$, as specified in Fig. 5. The QDT regions of the last two ${ }^{3} P^{o}$ target states are found to overlap with each other and are therefore set as degenerate. $\Omega(D R)$ consists of a large number of peaks corresponding to the detailed resonances, and although an extremely fine energy mesh is employed in the calculations of the $\Omega(\mathrm{DR})$ it cannot be assumed $a$ priori that the resolution is sufficient. However, the close agreement between the Maxwellian-averaged DR rate coefficients calculated with the resonanceaveraged $\langle\Omega(D R)\rangle$ and the $\Omega(D R)$ is an indication that most of the resonances have been resolved to yield an accurate integrated value.

Although the $\langle\Omega(D R)\rangle$ for $S$ II in Fig. 5 due to the $3 s 3 p^{3}\left({ }^{3} D^{o},{ }^{3} P^{o}\right)$ target thresholds are much smaller than those due to the higher thresholds $3 s 3 p^{3}\left({ }^{3} S^{o}\right)$, $3 p^{2} 3 p 3 d\left({ }^{3} D^{0},{ }^{3} P^{0}\right)$, since the corresponding $A$ values are one to two orders of magnitude smaller, the contribution to the high-energy recombination from these states is greater because of the exponentially decreasing Maxwellian distribution of electrons. This is also a general feature of the calculations: while all channels belonging to the

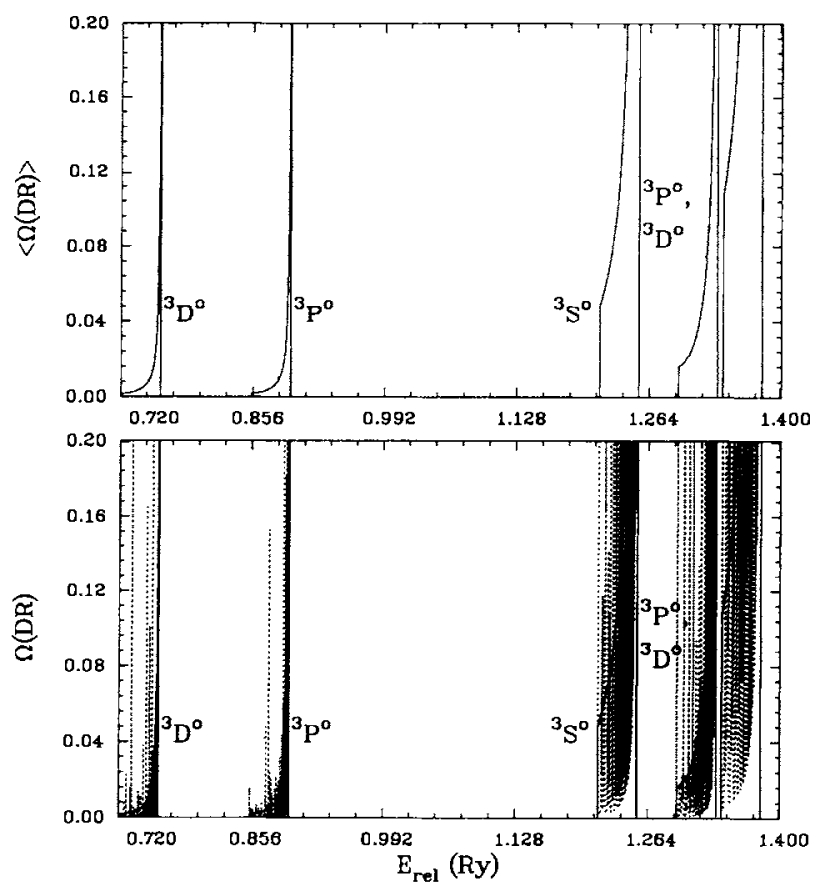

FIG. 5. Averaged (top) and detailed (bottom) DR collision strengths for the recombined ion S II in the entire energy range covered by the DR calculations. The DR calculations start at $v=10.0$ of each Rydberg series belonging to excited thresholds of the target ion S III. Note that the individual DR resonances, shown in expanded detail in Fig. 4, appear simply as vertical lines on the above scale.

target thresholds are included, the higher thresholds contribute much less than the very lowest thresholds in the target ion. This fact turns out to be advantageous in minimizing the uncertainties in the calculations due to overlapping QDT regions or the effect of strongly closed channels, all of which affect the higher, more closed space, thresholds more than the lower ones. In Fig. 5, for example, the lower two thresholds are separated from the higher ones by at least about $0.5 \mathrm{Ry}$, which ensures that the relatively larger $\Omega(D R)$ at higher energies will only contribute to the effective recombination rate at high temperatures $T>10^{5} \mathrm{~K}$.

At some of the higher thresholds the $\langle\Omega(D R)\rangle$ contains certain features that are attributable to the strongly closed channels belonging to thresholds higher than the one involved in the Gailitis averaging over the weakly closed channels. Again, the fact that the rate coefficients calculated from the detailed and the averaged $\Omega(D R)$ agree with each other indicates that the interference from the strongly closed channels does not lead to significant loss of accuracy in the case concerned. However, in general if the resonances from higher thresholds are sufficiently broad so as to attenuate to a large extent the narrow, densely packed resonances due to weakly closed channels in the QDT region, then we may only use the detailed $\Omega(D R)$ in our calculations. It may also be possible to renormalize the $\&$ matrix to eliminate the effect of the strongly closed channels, taking account of unitarity 
as it pertains to loss of flux in those channels. Some work is in progress along these lines.

\section{DR collision strengths for $e+S i X \rightarrow S i I X$}

Calculation of $\Omega$ (DR) for highly charged ions in the close-coupling approximation illustrates some different aspects than for lower ionization stages. As $z$ increases, the target thresholds within the same complex with $\Delta n=0$ may tend to become degenerate, and in calculating the resonance-averaged $\langle\Omega(D R)\rangle$ we may treat these thresholds as such. The procedure should be satisfactory for the higher thresholds in high-z ions. Figure 6 displays the $\langle\Omega(D R)\rangle$ for Si IX where, as in other B-like ions, we consider the three target thresholds $2 s 2 p^{2}\left({ }^{2} D,{ }^{2} S,{ }^{2} P\right)$ and set degenerate the latter two. Since $\Delta E\left({ }^{2} S-{ }^{2} P\right) \approx 0.2$ Ry, whereas $\Delta E\left({ }^{2} D-{ }^{2} S,{ }^{2} P\right) \approx 1.0 \mathrm{Ry}$, we expect no significant loss of accuracy due to the higher, degenerate thresholds, particularly given two facts: (i) we have not considered the fine structure in this work, and (ii) the entire DR contribution to the total $e+$ ion recombination becomes relatively smaller with increasing $z$ (discussed further in the next section). An additional independent check is provided by the $\Omega(E I E)$ at the thresholds, and the peak values of $(\Omega(D R)\rangle$, in accordance with Eq. (17) and as given in Table III. In the case of Si IX, the peak values of $\Omega(D R)$ and the $\langle\Omega(D R)\rangle$ at the degenerate ${ }^{2} S,{ }^{2} P$ thresholds is approximately equal to the sum of the $\Omega(\mathrm{EIE}): \quad \Omega\left({ }^{2} P^{o}-{ }^{2} S\right)+\Omega\left({ }^{2} P^{o}-{ }^{2} P\right)$. (All three sets of values with $I P E R T=0,1,2$ agree well with one another and the DR values.)

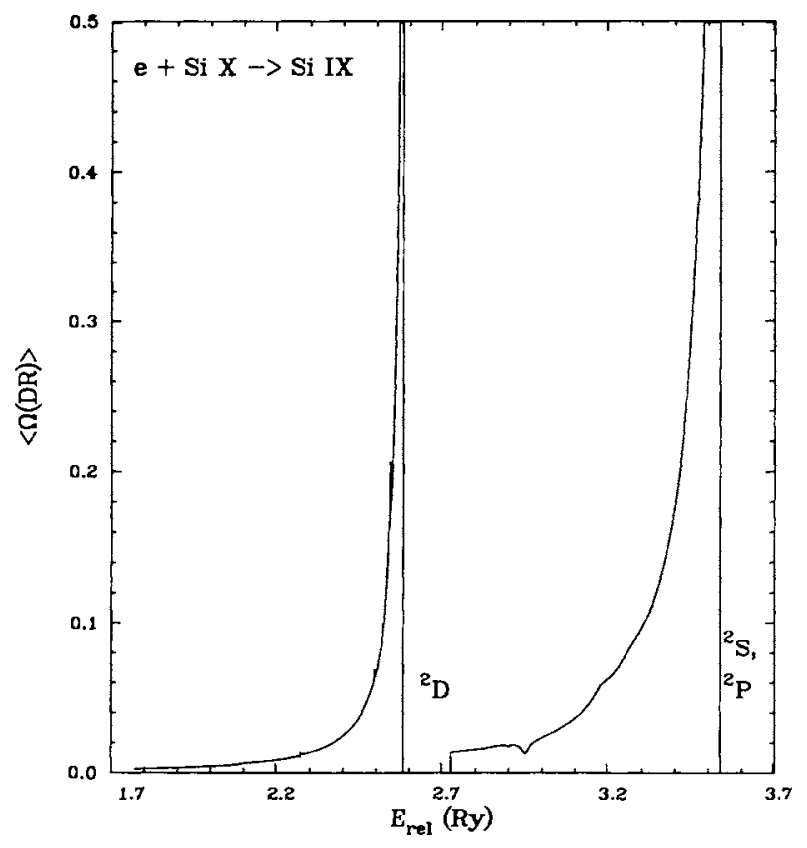

FIG. 6. The resonance-averaged $D R$ collision strength $\langle\Omega(D R)\rangle$ with $v \geq 10.0$ for each Rydberg series of recombined ion Si IX where the two closely spaced Si X target states ${ }^{2} S$ and ${ }^{2} \boldsymbol{P}$ are treated as degenerate. The small features superimposed on the averaged curve are due to strongly closed channels from higher target thresholds.
There are a few small features superimposed on the $\langle\Omega(D R)\rangle$ owing to the strongly closed channels as discussed in the preceding section, but these would clearly have negligible effect on the effective values. This is borne out by the good agreement between the Maxwellian-integrated rate coefficients obtained with $\Omega(D R)$ and $\langle\Omega(D R)\rangle$.

\section{Total recombination rate coefficients}

Maxwellian-averaged total $e+$ ion recombination rate coefficients, denoted hereafter as $\alpha_{R}(T)$, are calculated from the detailed photoionization cross sections in the low-energy region corresponding to low- $n$, and the highenergy region corresponding to the high- $n$ states of the recombined ion. Results are discussed first for individual examples Si IX, C II, and S II, and later for the carbon isoelectronic sequence. The general pattern of the $\alpha_{R}(T)$ is the same for all ions, as seen in the examples below. The $\alpha_{R}(T)$ decreases with temperature until a minimum is reached, and then with the onset of enhanced contributions from recombinations to high- $n$ autoionizing states (primarily DR), it exhibits a high-temperature bump followed by a second monotonic decrease. In addition, for some of the atomic systems the rate is enhanced, in the shape of a low-temperature bump, due to the autoionizing resonances in the photoionization cross sections of the low- $n$ states.

\section{Si $I X$}

Figure 7 shows the results for Si IX. The solid curve represents the total rate coefficient, while the dot-chained curve represents the low- $n(v \leq 10.0)$ contribution and the

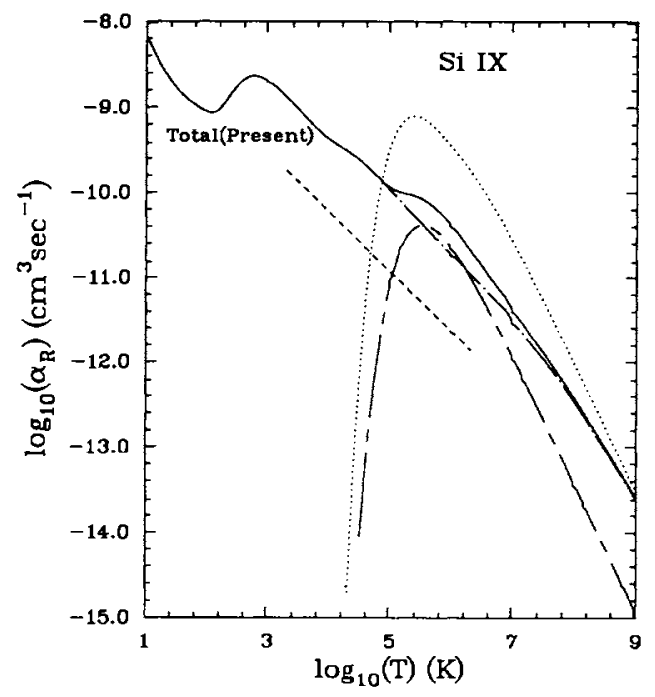

FIG. 7. Recombination rate coefficients for $\mathrm{SiX}+e \rightarrow \mathrm{Si}$ IX. The solid curve represents the present total rate coefficient, while the dot-chained curve represents the low-n contribution and the dash-chained curve represents high- $n$ contributions. Comparison is made with $R \mathbf{R}$ rate coefficients (dashed curve) of Ref. [24] and high-T DR rate coefficients (dotted curve) of Ref. [25]. 
dashed-chain curve represents the high-n $(10.0<v \leq \infty)$ contribution in the present calculations. As shown in the figure caption, comparison has been made with previously available results for RR [24] (dashed curve) and DR [25] (dotted curve) rate coefficients.

A number of features may be noted. A prominent bump in the low-temperature region can be seen. In general the autoionizing resonances in the photoionization cross sections of equivalent electron states cause the lowtemperature hump (see Fig. 2 and Table IV). (Some aspects of the recombination curve related to $z$ are discussed in the section on the carbon sequence ions, in particular the low-temperature bump.) The previous RR rate coefficients by Aldrovandi and Pequignot [24] (hereafter AP) underestimate the effective recombination by a large amount at low temperatures. However, the addition of low-temperature DR contribution to the RR should bring the values closer to the present results. The present calculations for the DR contribution from the $10.0<v \leq \infty$ range are considerably lower than the previous results [25]. In any case the high-energy (temperature) DR bump is itself rather small relative to the total. On the other hand, not only is the total recombination due predominantly to the $v \leq 10.0$ states, their highenergy (temperature) component is also quite appreciable (with the exception of a narrow temperature range $T \approx 1.5 \times 10^{5} \mathrm{~K}$, where DR is slightly larger).

\section{2. $C I I$}

Figure 8 presents $\alpha_{R}(T)$ for C II. The present results agree fairly well with the RR values of AP [24] at low temperatures, $T<10^{3}$. At higher temperatures, $T \approx 10^{4}$ $K$, the present results are lower than the low-temperature

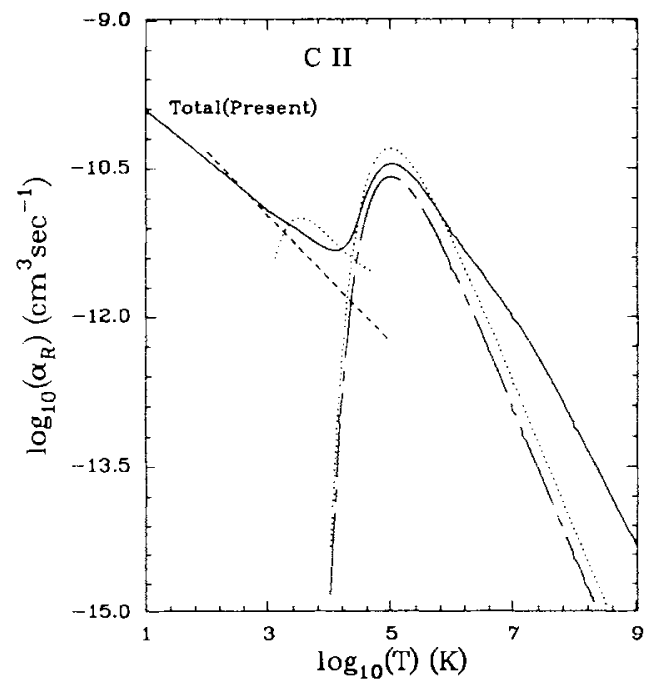

FIG. 8. Recombination rate coefficients for $\mathrm{C}$ III $+e \rightarrow \mathrm{C}$ II. The solid curve represents the present total rate coefficient and the dashed-chained curve represents the high- $n$ contribution alone. Comparison is made with $\mathrm{RR}$ rate coefficients (dashed curve) of Ref. [24], low-T DR rate coefficients (dotted curve) of Ref. [2], and high-T DR rate coefficients (dotted curve) of Ref. [25].
DR rate coefficients of Nussbaumer and Storey [2] (hereafter NS). Thus at $T \approx 10^{4} \mathrm{~K}$, the sum of the previous $\mathrm{RR}$ and the low-temperature $\mathrm{DR}$ rates overestimates the recombination rate compared to the present one. Around the high-temperature peak in DR, there appears to be good agreement with the earlier DR calculations of Jacobs et al. [25]. However, in the highest-temperature range, $T>10^{6.5} \mathrm{~K}$, the present rates are considerably higher than the earlier DR values, primarily due to the low- $n$ recombination component of the total, and not due to DR (since the present DR rate coefficients are in agreement with those of Jacobs et al. [25]). The hightemperature discrepancy is most probably due to the high-energy part of the photoionization cross sections of excited states. The PEC-type resonances discussed by $\mathrm{Yu}$ and Seaton [23] might also play an important role in high-energy (temperature) recombination for C II.

\section{3. $S_{I I}$}

Figure 9 shows the present $\alpha_{R}(T)$ for S II. In the lowtemperature region, $T<10^{4} \mathrm{~K}$, we find significant disagreement with the earlier $R R$ results of AP [24], which appear to overestimate and then underestimate the recombination rate as shown. The present values near the high-temperature bump agree well in all of the hightemperature region with the earlier DR calculations of Jacobs et al. [25] whereas the rate coefficients of Badnell [26] lie above the present values. In order to preserve clarity in Fig. 9, the present results for the low- $n$ and the high- $n$ (DR) regions are not shown separately.

\section{Carbon sequence ions}

Figure 10 presents the results for several $\mathrm{C}$ sequence ions and shows the main trend in the total recombination

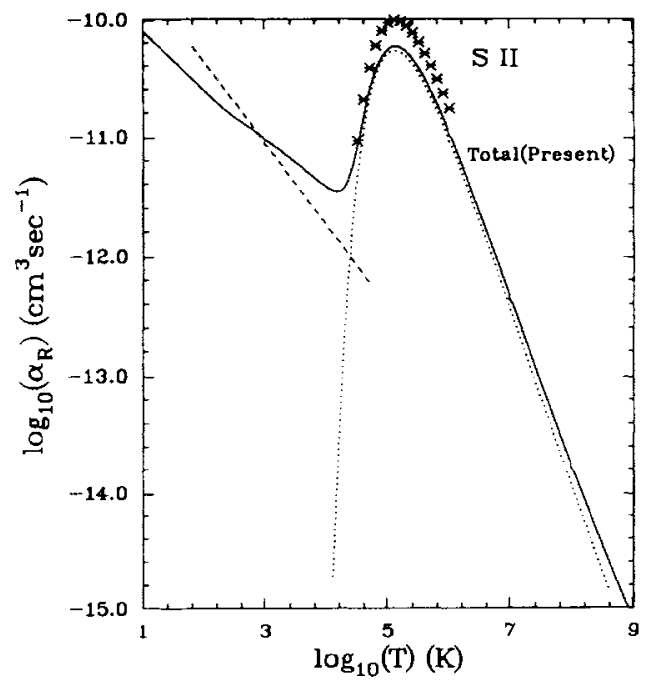

FIG. 9. Recombination rate coefficients for $\mathrm{S}$ III $+e \rightarrow \mathrm{S}_{\mathrm{II}}$. The solid curve represents the present total rate coefficient. Comparison is made with $\mathbf{R R}$ rate coefficients (dashed curve) of Ref. [24], high-T DR rate coefficients of Ref. [25] (dotted curve) and of Ref. [26] (asterisks). 


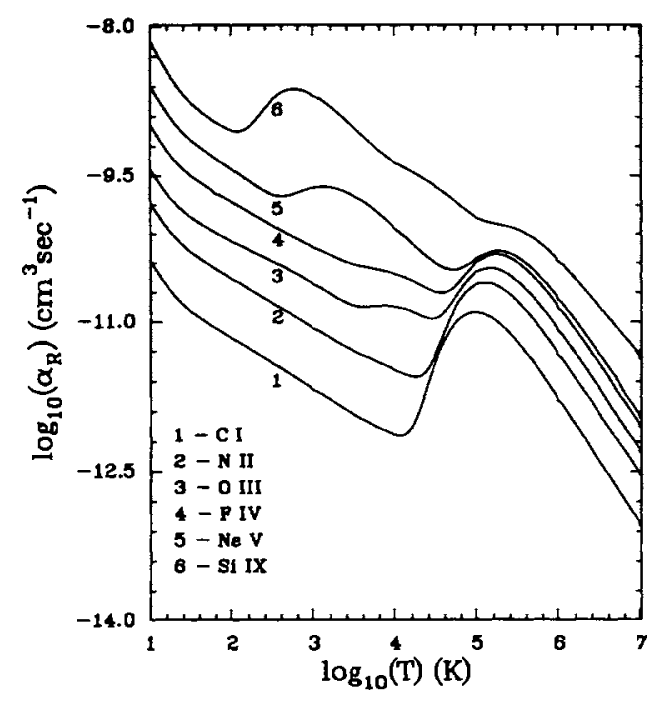

FIG. 10 Total recombination rate coefficients $\alpha_{R}(T)$ for ions in the carbon isoelectronic sequence. The figure illustrates that electron-ion recombination through the continuua increases with $z$ relative to the recombination through the autoionizing states.

rate coefficient $\alpha_{R}(T)$ vs $z$ : the low-energy (temperature) recombination increases while the high-energy (temperature) component decreases in relative magnitude. (In conventional terms one could refer to it as the increase of RR over DR with z.) The general pattern shown in Fig. 10 is due to the interplay of the following main factors.

(1) The number of bound states of the recombined ion in the low- $n$ region increases rapidly with ion charge (Table IV), and a large number of such states contribute to the enhancement which occurs at relatively lower energies (the photoionization cross sections decrease with energy as $\sigma_{\mathrm{PI}} \approx \omega^{-3}$ ).

(2) While the autoionization rate is independent of $z$, the total contribution from the high- $n$ states, through the autoionizing states, saturates with $z$ and becomes relatively less important.

(3) The contribution of the low-n states continues to higher energies and temperatures, as seen by the relative enhancement of the background over the hightemperature bump in $\alpha_{R}(T)$. A simple way to look at it is that the recombination through the continuum increases while that through the autoionizing states decreases with $z$. The phenomenon has been experimentally observed in studies of very highly charged ions [27], but to our knowledge has not been studied for lighter systems such as are under consideration herein. It is of some interest therefore to note that the high- $n$ DR may not be dominant or even relatively large for highly charged ions.

(4) The low- $n$ DR contribution, however, is due to the low-lying autoionizing states close to the ionization threshold and may be significant even for highly charged ions, as seen in the low-temperature bump in $\alpha_{R}(T)$. There are two features associated with the lowtemperature bump: its position depends on the energies of the autoionizing resonances relative to the ionization threshold, and its size may be significantly enhanced in the low-temperature region if the resonances are very close to the threshold, owing to the Maxwellian in $\alpha_{R}(T)$, Eq. (3), that contains a $T^{-3 / 2}$ factor. For $O$ III the lowtemperature bump begins as a small rise, which becomes much more pronounced for $\mathrm{Ne} \mathrm{V}$ at somewhat lower temperatures, and still larger for $\mathrm{Si}$ IX at even lower temperatures. However, preliminary results for two other ions in the sequence, $\mathrm{Mg}$ VII and SXI, show that the $\alpha_{R}(T)$ for the higher-z ions may overlap with that of other ions in the low-temperature region, if the bump is absent. For example, we find that for $\mathrm{Mg}$ VII the low-temperature values are lower than for $\mathrm{Ne} \mathrm{V}$ in the region covered by the bump in NeV. But, the trend for all ions in the sequence in the high-temperature region remains the same as shown in Fig. 10, i.e., the $\alpha_{R}(T)$ converge as a function of $z$.

(5) While the presence or the position and size of the low-energy bump may differ considerably, as discussed above, the temperature range of the high-temperature bump in $\alpha_{R}(T)$ appears to be relatively close for all the carbon ions, progressing slowly to higher $T$ with $z$.

(6) The possibility of additional bumps at very high temperatures, associated with inner shell ionization and consequent recombination, e.g., via Auger transitions, may not be ruled out.

The effect of $\Delta n \neq 0$ transitions in the DR rate coefficients may be significant and is being investigated by including additional states of the higher $n$ complexes.

\section{CONCLUSION}

The method presented appears to be quite general for accurate computation of unified $e+$ ion recombination rate coefficients. The present treatment enables precise and consistent computation of recombination through the continuum and the autoionization, while displaying the regimes where one or the other may be more important. Calculations have been completed, or are continuing, for several atoms and ions other than the ones reported here. The following points, however, need to be noted.

Fine structure has not, yet, been considered in the present formulation. In principle there is no difficulty in incorporating fine structure within the CC framework. This may be accomplished in two ways: (i) an algebraic transformation of the scattering matrices to a pair coupling scheme such as has been employed in a large number of electron-ion scattering calculations [28], (ii) BreitPauli calculations that include the fine structure in an $a b$ initio manner. Both of these extensions are in progress. The effect of intermediate coupling depends on the ion charge and should be quite small for low-charge ions, but may be considerable for highly charged ions [29].

The CC method should be particularly useful in the calculation of total recombination rates for complex atomic systems (e.g., Fe II), where one needs to consider a very large number of states and corresponding autoionization and radiative transitions that are coupled together. Also, the recombination calculations are not likely to suffer from the problem of the convergence of the CC expansion that is common for electron-ion scattering, since 
we consider relatively lower electron energies. It is difficult to see how the independent-processes and the isolated-resonance approximations, hitherto employed in recombination work, can deal with the inherently complex channel couplings, although one might expect such approximations to be valid for light or highly ionized systems [30]. The recent work on Fe II [31], for example, includes 83 coupled $L S$ terms dominated by target configurations $3 d^{6} 4 s, 3 d^{7}, 3 d^{6} 4 p$. Recombination calculations based on the new Fe II calculations are in progress.

Another topic of importance under study within the present framework is the effect of external plasma microfields on DR. There are several factors to consider: (i) it appears that while static external fields enhance the DR rate in laboratory experiments on singly ionized ions by several factors [32], the effect on multiply charged ions may be much less, as shown in the recent work on CIV where the effective DR rate is estimated to increase at most by $40 \%$ [33]; (ii) we have shown that the importance of DR itself, relative to the continuum contribution in the total $\alpha_{R}(T)$, decreases with $z$ due to the dominance of the nuclear over the electronic potential; for example, the Si IX recombination rate coefficients are unlikely to be affected significantly by external fields since the high- $n$ component, subject to the field effects, is smaller than the low- $n$ and the continuum components in nearly the entire temperature range; (iii) the quantitative effect of plasma microfields on autoionization in specific systems is unknown; Stark-field and electron impact ionization of the high- $n$ autoionizing states also need to be taken into account (some discussion in connection with bound states is provided by the recent work by Hummer and Mihalas in their equation-of-state formalism for the Opacity Project
[34]). As the present rate coefficients do not take account of field effects in the high-n part, the uncertainty for low ionization stages is higher at plasma densities where the fields may be appreciable. However, it should be emphasized that since the effective $\alpha_{R}(T)$ is a combination of continuum as well as the autoionization part, the overall uncertainty would generally be smaller in the total recombination rate than found in DR alone. In optically thin astrophysical plasmas (e.g., in the interstellar medium, H II regions, etc. with $N_{e}<10^{6} \mathrm{~cm}^{-3}$ ), the present rates should be quite accurate, to within the usual uncertainties in CC calculations, i.e., 10-20\%. This estimate is based on the better-known uncertainties in the two related and essential components of the present $\mathrm{CC}$ calculations for recombination: photoionization cross sections and the EIE collision strengths. We expect the $\Omega(D R)$ to be of the same accuracy as low-energy $\Omega$ (EIE) in the $\mathrm{CC}$ approximation, i.e., $\approx 10 \%$. An uncertainty of similar magnitude is usually estimated in the CC photoionization calculations.

For plasma modeling one needs the total $\alpha_{R}(T)$ and a tabulation of these for a number of atoms and ions (including several not described here) will be published in the near future.

\section{ACKNOWLEDGMENTS}

This work was supported in part by Grant No. NAGW-3315 from the NASA LTSA program and by Grant No. PHY-9115057 from the NSF. The computational work was carried out on the Cray Y-MP computer at the Ohio Supercomputer Center in Columbus, Ohio.
[1] M. R. Flannery, in Adv. At. Mol. Phys. 32, 117 (1993); Y. Hahn, Phys. Rep. 166, 195 (1988).

[2] H. Nussbaumer and P. J. Storey, Astron. Astrophys. 126, 75 (1983); Astron. Astrophys. Suppl. 69, 123 (1987).

[3] D. E. Osterbrock, Astrophysics of Gaseous Nebulae and Active Galactic Nuclei (University Science Books, Mill Valley, CA, 1989).

[4] S. N. Nahar and A. K. Pradhan, Phys. Rev. A 44, 2935 (1991), referred to as NP1; J. Phys. B 26, 1109 (1993).

[5] S. N. Nahar and A. K. Pradhan, Phys. Rev. Lett. 68, 1488 (1992), referred to as NP2.

[6] M. J. Seaton, J. Phys. B 20, 6363 (1987).

[7] K. A. Berrington, P. G. Burke, K. Butler, M. J. Seaton, P. J. Storey, K. T. Taylor, and Y. Yan, J. Phys. B 20, 6379 (1987).

[8] R. H. Bell and M. J. Seaton, J. Phys. B 18, 1589 (1985).

[9] G. Alber, J. Cooper, and A. R. P. Rau, Phys. Rev. A 30, 2845 (1984).

[10] S. L. Haan and V. L. Jacobs, Phys. Rev. A 40, 80 (1989).

[11] M. Gailitis, Zh. Eksp. Teor. Fiz. 44, 1974 (1963) [Sov. Phys. JETP 17, 1328 (1963)].

[12] P. C. W. Davies and M. J. Seaton, J. Phys. B 2, 757 (1969).

[13] M. J. Seaton, J. Phys. B 2, 5 (1969).

[14] A. K. Pradhan, Phys. Rev. Lett. 47, 79 (1981).

[15] A. K. Pradhan and M. J. Seaton, J. Phys. B 18, 1631
(1985).

[16] W. Eissner, M. Jones, and H. Nussbaumer, Comput. Phys. Commun. 8, 270 (1974).

[17] M. J. Seaton, Y. Yu, D. Mihalas, and A. K. Pradhan, Mon. Not. R. Astron. Soc. 266, 805 (1994).

[18] P. J. Storey and D. G. Hummer, Comput. Phys. Commun. 66, 129 (1991).

[19] D. Luo and A. K. Pradhan, J. Phys. B 22, 3377 (1989).

[20] M. J. Seaton, J. Phys. B 18, 2111 (1985).

[21] M. J. Seaton, Comput. Phys. Commun. 25, 87 (1982).

[22] S. T. Manson, Phys. Rev. A 46, 2939 (1992).

[23] Y. Yu and M. J. Seaton, J. Phys. B 20, 6409 (1987).

[24] S. M. V. Aldrovandi and D. Pequignot, Astron. Astrophys. 25, 137 (1973).

[25] J. M. Shull and M. V. Steenberg, Astrophys. J. Suppl. 48, 95 (1982); they fitted the DR rate coefficient curves from the values calculated by Jacobs et al. References are given in Shull and Steenberg's paper.

[26] N. R. Badnell, Astrophys. J. 379, 356 (1991).

[27] W. Spies, A. Muller, J. Linkemann, A. Frank, M. Wangner, C. Kozhuharov, B. Franzke, K. Beckert, F. Bosch, H. Eickhoff, M. Jung, O. Klepper, W. Konig, P. H. Mokler, R. Moshammer, F. Nolden, U. Schaaf, P. Spadtke, M. Steck, P. Zimmerer, N. Grun, W. Sheid, M. S. Pindzola, and N. R. Badnell, Phys. Rev. Lett. 69, 2768 
(1992).

[28] D. Luo and A. K. Pradhan, Phys. Rev. A 41, 165 (1990).

[29] N. R. Badnell, J. Phys. B 23, L565 (1990).

[30] M. S. Pindzola, N. R. Badnell, and D. C. Griffin, Phys. Rev. A 46, 5725 (1992).

[31] S. N. Nahar and A. K. Pradhan, J. Phys. B (to be published).
[32] D. S. Belic, G. H. Dunn, T. J. Morgan, D. W. Mueller, and C. Timmer, Phys. Rev. Lett. 50, 339 (1983).

[33] N. R. Badnell, M. S. Pindzola, W. J. Dickens, H. P. Summers, D. C. Griffin, and J. Lang, Astrophys. J. Lett. 407, L91 (1993).

[34] D. G. Hummer and D. Mihalas, Astrophys. J. 331, 794 (1988). 